\title{
An Efficient, Energy Stable Scheme for the Cahn-Hilliard-Brinkman System
}

\author{
Craig Collins $^{1}$, Jie Shen ${ }^{2}$ and Steven M. Wise ${ }^{1, *}$ \\ ${ }^{1}$ Department of Mathematics, University of Tennessee, Knoxville, TN 37912, USA. \\ 2 Department of Mathematics, Purdue University, West Lafayette, IN 47907, USA.
}

Received 17 December 2011; Accepted (in revised version) 13 April 2012

Communicated by Bo Li

Available online 21 September 2012

\begin{abstract}
We present an unconditionally energy stable and uniquely solvable finite difference scheme for the Cahn-Hilliard-Brinkman (CHB) system, which is comprised of a Cahn-Hilliard-type diffusion equation and a generalized Brinkman equation modeling fluid flow. The CHB system is a generalization of the Cahn-Hilliard-Stokes model and describes two phase very viscous flows in porous media. The scheme is based on a convex splitting of the discrete $\mathrm{CH}$ energy and is semi-implicit. The equations at the implicit time level are nonlinear, but we prove that they represent the gradient of a strictly convex functional and are therefore uniquely solvable, regardless of time step size. Owing to energy stability, we show that the scheme is stable in the time and space discrete $\ell^{\infty}\left(0, T ; H_{h}^{1}\right)$ and $\ell^{2}\left(0, T ; H_{h}^{2}\right)$ norms. We also present an efficient, practical nonlinear multigrid method - comprised of a standard FAS method for the Cahn-Hilliard part, and a method based on the Vanka smoothing strategy for the Brinkman part - for solving these equations. In particular, we provide evidence that the solver has nearly optimal complexity in typical situations. The solver is applied to simulate spinodal decomposition of a viscous fluid in a porous medium, as well as to the more general problems of buoyancy- and boundary-driven flows.
\end{abstract}

AMS subject classifications: 65M06, 65M12, 65M55, 76T99

Key words: Cahn-Hilliard equation, Stokes equations, Brinkman equation, finite difference methods, nonlinear multigrid, convex splitting, energy stability.

\section{Introduction}

\subsection{Problem definition and background}

Consider the Ginzburg-Landau energy of a binary fluid with constant, uniform mass density [5]

*Corresponding author. Email addresses: craig. collins@math .utk. edu (C. Collins), shen@math.purdue.edu (J. Shen), swise@math.utk.edu (S. M. Wise) 


$$
E(\phi)=\int_{\Omega}\left\{\frac{1}{4 \epsilon}\left(\phi^{2}-1\right)^{2}+\frac{\epsilon}{2}|\nabla \phi|^{2}\right\} d \mathbf{x},
$$

where $\Omega \subset \mathbb{R}^{D}, D=2$ or $3, \phi: \Omega \rightarrow \mathbb{R}$ is the concentration field, and $\epsilon$ is a positive constant. The phase equilibria are represented by the pure fluids $\phi= \pm 1$. The dynamical CahnHilliard-Brinkman equations we consider are

$$
\begin{aligned}
& \partial_{t} \phi=\epsilon \nabla \cdot(M(\phi) \nabla \mu)-\nabla \cdot(\phi \mathbf{u}), \\
& -\nabla \cdot[v(\phi) \mathrm{D}(\mathbf{u})]+\eta(\phi) \mathbf{u}=-\nabla p-\gamma \phi \nabla \mu, \\
& \nabla \cdot \mathbf{u}=0,
\end{aligned}
$$

where $M(\phi)$ is a mobility that incorporates the Peclet number; $\mu$ is the chemical potential

$$
\mu:=\delta_{\phi} E=\frac{1}{\epsilon}\left(\phi^{3}-\phi\right)-\epsilon \Delta \phi ;
$$

$\gamma>0$ is a surface tension parameter; $\mathbf{u}$ is the fluid velocity; $p$ is a pressure; $v(\cdot)>0$ is the fluid viscosity; $\eta(\cdot)>0$ is the fluid permeability; and $\mathrm{D}(\mathbf{u})=\nabla \mathbf{u}+(\nabla \mathbf{u})^{T}$.

We assume that $M, v, \eta \in C^{\infty}$, and $M(x) \geq M_{0}>0, \eta(x) \geq \eta_{0}>0$, and $v(x) \geq v_{0}>0$, for all $x \in \mathbb{R}$. For example, we shall frequently use a regularized degenerate mobility of the form

$$
M(\phi)=\frac{1}{\mathrm{Pe}} \sqrt{(1+\phi)^{2}(1-\phi)^{2}+\epsilon^{2}} \geq \frac{\epsilon}{\mathrm{Pe}}>0,
$$

where $\mathrm{Pe}>0$ is the Peclet number, which might be dependent upon $\epsilon$. We assume that the system (1.2)-(1.4) is supplemented with the boundary conditions $\left.\mathbf{u}\right|_{\partial \Omega}=0$, and $\left.\partial_{n} \phi\right|_{\partial \Omega}=\left.\partial_{n} \mu\right|_{\partial \Omega}=0$. The latter conditions represent local thermodynamic equilibrium on the boundary. With this set of boundary conditions, the system (1.2)-(1.4) is mass conservative and energy dissipative, and the dissipation rate is readily found to be $[8,14,15,17]$

$$
d_{t} E=-\epsilon(M(\phi) \nabla \mu, \nabla \mu)_{L^{2}}-\frac{1}{\gamma}(\eta(\phi) \mathbf{u}, \mathbf{u})_{L^{2}}-\frac{1}{2 \gamma}(\nu(\phi) \mathrm{D}(\mathbf{u}), \mathrm{D}(\mathbf{u}))_{L^{2}} \leq 0 .
$$

Eq. (1.3) is a generalized Brinkman equation [2] that incorporates a diffuse interface surface tension force. The Cahn-Hilliard-Brinkman (CHB) system (1.2)-(1.4) was recently proposed as a model for phase separation and coarsening of a binary fluid in a Brinkman porous medium [18]. The authors showed the existence of a logarithmically slow coarsening regime that arises when the phase domains are comparable to the average pore size. The system (1.2)-(1.4) is also closely related to models of tumor growth [20,33,34] which include an additional mass source for volumetric growth. When the surface tension vanishes, i.e., $\gamma=0$, the CHB system reduces to the Cahn-Hilliard equation [3]. A generalized Stokes equation is obtained when $\eta \equiv 0$ in (1.3). The system (1.2)-(1.4) is a simplified version of the model derived by Lee et al. [14,15], which they used to describe gravity-driven, density-mismatched, two-phase flow. We remark that the CHB system 
is closely related to the Cahn-Hilliard-Navier-Stokes systems studied by $[8,12-17,22,24]$ and many others. We will discuss this connection a bit more at the conclusion of the paper.

Note that we specifically use the surface tension force formulation $\mathbf{f}=-\gamma \phi \nabla \mu$, which was used by Feng [8] and others. This will be a vital point for the solvability of the fully discrete scheme. However, at the continuous level, the form we use is equivalent to others. For example, introducing a new pressure $\tilde{p}:=p+\gamma \phi \mu$, we find

$$
-\nabla \cdot[v(\phi) \mathrm{D}(\mathbf{u})]+\eta(\phi) \mathbf{u}=-\nabla \tilde{p}-\gamma \mu \nabla \phi,
$$

which is the form used in $[18,22]$ and elsewhere.

In this paper, we propose and analyze a convex-splitting scheme for the CHB system (1.2)-(1.4). We prove that the scheme is unconditionally stable and solvable, and we present a practical and efficient multigrid method for solving the nonlinear system at each time step.

\subsection{A convex splitting scheme in time}

In this section we describe a time-discrete scheme for the $\mathrm{CHB}$ system. The calculations here are meant to motivate those for the fully discrete scheme that we exhibit in later sections. The scheme is based on a convex splitting approach $[1,6,7,27]$ that we have used in earlier works $[10,21,23,28,29,35]$. There are two important properties that convex splitting schemes generally inherit, unconditional energy stability and unconditional unique solvability $[7,35]$. However, convex splitting schemes are typically nonlinear, which presents a challenge with respect to practical implementation. Later we will show that nearly optimally efficient algorithms are available for solving such nonlinear schemes.

Notice that the energy (1.1) can be written as the difference of two purely convex pieces $E_{c}-E_{e}$ with, for example,

$$
E_{c}=\int_{\Omega}\left\{\frac{1}{4 \epsilon} \phi^{4}+\frac{1}{4 \epsilon}+\frac{\epsilon}{2}|\nabla \phi|^{2}\right\} d \mathbf{x}, \quad E_{e}=\int_{\Omega} \frac{1}{2 \epsilon} \phi^{2} d \mathbf{x} .
$$

The key idea of the convex splitting schemes is to treat $E_{c}$ implicitly, while treating $E_{e}$ explicitly in a time-discrete scheme. Hence, a first-order semi-implicit, convex splitting scheme is as follows:

$$
\begin{aligned}
& \phi^{k+1}-\phi^{k}=s \nabla \cdot\left(\epsilon M\left(\phi^{k}\right) \nabla \mu^{k+1}-\phi^{k} \mathbf{u}^{k+1}\right), \\
& \mu^{k+1}:=\delta_{\phi} E_{c}\left(\phi^{k+1}\right)-\delta_{\phi} E_{e}\left(\phi^{k}\right)=\frac{1}{\epsilon}\left(\phi^{k+1}\right)^{3}-\frac{1}{\epsilon} \phi^{k}-\epsilon \Delta \phi^{k+1}, \\
& -\nabla \cdot\left[\nu\left(\phi^{k}\right) \mathrm{D}\left(\mathbf{u}^{k+1}\right)\right]+\eta\left(\phi^{k}\right) \mathbf{u}^{k+1}=-\nabla p^{k+1}-\gamma \phi^{k} \nabla \mu^{k+1}, \\
& \nabla \cdot \mathbf{u}^{k+1}=0,
\end{aligned}
$$


where $s>0$ is the time step size, with the boundary conditions

$$
\left.\partial_{n} \phi^{k+1}\right|_{\partial \Omega}=\left.\partial_{n} \mu^{k+1}\right|_{\partial \Omega}=0,\left.\quad \mathbf{u}\right|_{\partial \Omega}=\mathbf{0} .
$$

Theorem 1.1. The scheme (1.10)-(1.13) is uniquely solvable for any time step $s>0$; mass conservative, i.e.,

$$
\left(\phi^{k+1}, 1\right)_{L^{2}}=\left(\phi^{k}, 1\right)_{L^{2}}, \quad \forall k \geq 0 ;
$$

and unconditionally energy stable, i.e.,

$$
E\left(\phi^{k+1}\right) \leq E\left(\phi^{k}\right), \quad \forall k \geq 0,
$$

for any time step $s>0$.

Proof. For brevity, we omit the proof for the unique solvability here, but refer to the proof in Section 2 for the fully discrete scheme, which is based on tools from convex analysis.

Integrating (1.10) over $\Omega$, thanks to (1.14), we derive immediately $\left(\phi^{k+1}, 1\right)_{L^{2}}=\left(\phi^{k}, 1\right)_{L^{2}}$. Next, we take the inner products of (1.10) with $\mu^{k+1}$, of (1.11) with $-\left(\phi^{k+1}-\phi^{k}\right)$ and of (1.12) with $(s / \gamma) \mathbf{u}^{k+1}$, sum up the three relations and integrate by parts, we find that

$$
\begin{aligned}
E\left(\phi^{k+1}\right)-E\left(\phi^{k}\right)= & -s \epsilon\left(M\left(\phi^{k}\right) \nabla \mu^{k+1}, \nabla \mu^{k+1}\right)_{L^{2}}-\frac{s}{\gamma}\left(\eta\left(\phi^{k}\right) \mathbf{u}^{k+1}, \mathbf{u}^{k+1}\right)_{L^{2}} \\
& -\frac{s}{2 \gamma}\left(v\left(\phi^{k}\right) \mathrm{D}\left(\mathbf{u}^{k+1}\right), \mathrm{D}\left(\mathbf{u}^{k+1}\right)\right)_{L^{2}} \\
& -\frac{1}{4 \epsilon}\left\|\left(\phi^{k+1}\right)^{2}-\left(\phi^{k}\right)^{2}\right\|_{L^{2}}^{2}-\frac{1}{2 \epsilon}\left\|\phi^{k+1}\left(\phi^{k+1}-\phi^{k}\right)\right\|_{L^{2}}^{2} \\
& -\frac{1}{2 \epsilon}\left\|\phi^{k+1}-\phi^{k}\right\|_{L^{2}}^{2}-\frac{\epsilon}{2}\left\|\nabla \phi^{k+1}-\nabla \phi^{k}\right\|_{L^{2}}^{2} .
\end{aligned}
$$

Thanks to the non-positivity of all terms on the right-hand-side, this implies

$$
E\left(\phi^{k+1}\right) \leq E\left(\phi^{k}\right)
$$

regardless of the time step size $s$.

In the remainder of the paper we demonstrate that the convex-splitting framework outlined in this section has an analog in the spatial discrete setting, specifically using difference operators. In particular, the fully discrete scheme that we propose later for the CHB system will be shown to be unconditionally energy stable and unconditionally uniquely solvable. We also develop a practical and efficient nonlinear solver for the scheme in this paper. In Section 2 we present the main results of our analyses, including the unique solvability and discrete-energy stability of our scheme. In Section 3 we present evidence that our multigrid solver, described later in Appendix B, is of nearly optimal complexity. We also provide a convergence test for the scheme, showing that it 
is first order accurate in time and second order accurate in space. In Section 4 we use the scheme to simulate spinodal decomposition, as modeled by the CHB system (1.2)-(1.4). We also use our scheme to simulate two cases of driven flow. We give some concluding remarks and suggest some future work in Section 5. In the first appendix, Appendix A, we describe some details of the staggered grid finite difference space discretization that we employ in the scheme. In the second appendix, Appendix B, we give some details of the efficient nonlinear multigrid method for solving the semi-implicit scheme in time.

\section{Fully discrete scheme and its stability analysis}

In this section we describe and analyze our staggered grid finite difference scheme for the CHB system (1.2)-(1.4). We refer the reader to Appendix A for a description of our notation and for some useful results, such as the various summation-by-parts formulae that are commonly used in the analyses.

\subsection{The fully discrete convex splitting scheme}

We begin by defining a fully discrete energy that is consistent with the continuous space energy (1.1). In particular, define the discrete energy $F: \mathcal{C}_{\bar{m} \times \bar{n}} \rightarrow \mathbb{R}$ to be

$$
F(\phi):=\frac{1}{4 \epsilon}\|\phi\|_{4}^{4}+\frac{1}{4 \epsilon}-\frac{1}{2 \epsilon}\|\phi\|_{2}^{2}+\frac{\epsilon}{2}\left\|\nabla_{h} \phi\right\|_{2}^{2} .
$$

It is straightforward to show that if $\phi \in \mathcal{C}_{\bar{m} \times \bar{n}}$ and $\left.\mathbf{n} \cdot \nabla_{h} \phi\right|_{\partial \Omega}=0$, the energies

$$
F_{c}(\phi):=\frac{1}{4 \epsilon}\|\phi\|_{4}^{4}+\frac{1}{4 \epsilon}+\frac{\epsilon}{2}\left\|\nabla_{h} \phi\right\|_{2}^{2}, \quad F_{e}(\phi):=\frac{1}{2 \epsilon}\|\phi\|_{2}^{2}
$$

are convex. Hence $F$, as defined in (2.1), admits the convex splitting $F=F_{c}-F_{e}$. The gradients (discrete variations) of the respective energies are $\delta_{\phi} F_{c}=\frac{1}{\epsilon} \phi^{3}-\epsilon \Delta_{h} \phi$ and $\delta_{\phi} F_{e}=$ $\frac{1}{\epsilon} \phi$.

The fully-discrete scheme for the Cahn-Hilliard-Brinkman system is the following: given $\phi^{k} \in \mathcal{C}_{\bar{m} \times \bar{n}}$, find the grid functions $\phi^{k+1}, \mu^{k+1}, p^{k+1} \in \mathcal{C}_{\bar{m} \times \bar{n}}, u^{k+1} \in \mathcal{E}_{m \times n}^{\mathrm{ew}}$, and $v^{k+1} \in$ $\mathcal{E}_{m \times n}^{\text {ns }}$ such that

$$
\begin{aligned}
& \phi^{k+1}-\phi^{k}= s \epsilon\left[d_{x}\left(M\left(A_{x} \phi^{k}\right) D_{x} \mu^{k+1}\right)+d_{y}\left(M\left(A_{y} \phi^{k}\right) D_{y} \mu^{k+1}\right)\right] \\
&-s\left[d_{x}\left(A_{x} \phi^{k} u^{k+1}\right)+d_{y}\left(A_{y} \phi^{k} v^{k+1}\right)\right], \\
& \mu^{k+1}:=\delta_{\phi} F_{c}\left(\phi^{k+1}\right)-\delta_{\phi} F_{e}\left(\phi^{k}\right)=\frac{1}{\epsilon}\left(\phi^{k+1}\right)^{3}-\frac{1}{\epsilon} \phi^{k}-\epsilon \Delta_{h} \phi^{k+1},
\end{aligned}
$$




$$
\begin{aligned}
-\gamma A_{x} \phi^{k} D_{x} \mu^{k+1}= & D_{x} p^{k+1}+\eta\left(A_{x} \phi^{k}\right) u^{k+1}-2 D_{x}\left(v\left(\phi^{k}\right) d_{x} u^{k+1}\right) \\
& -d_{y}\left\{v\left(\mathcal{A} \phi^{k}\right)\left(D_{y} u^{k+1}+D_{x} v^{k+1}\right)\right\}, \\
-\gamma A_{y} \phi^{k} D_{y} \mu^{k+1}= & D_{y} p^{k+1}+\eta\left(A_{y} \phi^{k}\right) v^{k+1}-2 D_{y}\left(v\left(\phi^{k}\right) d_{y} v^{k+1}\right) \\
& -d_{x}\left\{v\left(\mathcal{A} \phi^{k}\right)\left(D_{x} v^{k+1}+D_{y} u^{k+1}\right)\right\}, \\
d_{x} u^{k+1}+d_{y} v^{k+1}= & 0 .
\end{aligned}
$$

We enforce the solvability condition $\left(p^{k+1} \| \mathbf{1}\right)=0$ and the boundary conditions

$$
\left.\mathbf{n} \cdot \nabla_{h} \phi^{k+1}\right|_{\partial \Omega}=\left.\mathbf{n} \cdot \nabla_{h} \mu^{k+1}\right|_{\partial \Omega}=0,\left.\quad u^{k+1}\right|_{\partial \Omega}=\left.v^{k+1}\right|_{\partial \Omega}=0 .
$$

Once again, the reader is referred to Appendix A and [32,35] for a description of the finite difference notation used here.

In order to make the calculations that follow more readable, let us define

$$
\begin{array}{ll}
\phi^{\mathrm{ew}}:=A_{x} \phi^{k}, & \phi^{\mathrm{ns}}:=A_{y} \phi^{k} ; \\
M^{\mathrm{ew}}:=M\left(\phi^{\mathrm{ew}}\right), & M^{\mathrm{ns}}:=M\left(\phi^{\mathrm{ns}}\right) ; \\
\eta^{\mathrm{ew}}:=\eta\left(\phi^{\mathrm{ew}}\right), & \eta^{\mathrm{ns}}:=\eta\left(\phi^{\mathrm{ns}}\right) ; \\
v^{\mathrm{vc}}:=v\left(\mathcal{A} \phi^{k}\right), & v^{\mathrm{cc}}:=v\left(\phi^{k}\right) .
\end{array}
$$

We will use this notation throughout the remainder of the paper. Also, we point out that the scheme simplifies in the case of constant viscosity. For instance, if $v \equiv 1$, Eqs. (2.5) and (2.6) become

$$
\begin{aligned}
& -\gamma A_{x} \phi^{k} D_{x} \mu^{k+1}=D_{x} p^{k+1}+\eta^{\mathrm{ew}} u^{k+1}-D_{x}\left(d_{x} u^{k+1}\right)-d_{y}\left(D_{y} u^{k+1}\right), \\
& -\gamma A_{y} \phi^{k} D_{y} \mu^{k+1}=D_{y} p^{k+1}+\eta^{\mathrm{ns}} v^{k+1}-D_{y}\left(d_{y} v^{k+1}\right)-d_{x}\left(D_{x} v^{k+1}\right),
\end{aligned}
$$

upon using our simplified notation and the discrete divergence-free condition Eq. (2.7).

Now we perform some calculations on the discrete Brinkman equations (2.5)-(2.7) that will be used several times in the following sections. To simplify the notations, let us make the notation replacements $\mu^{k+1} \rightarrow \mu_{\alpha}, p^{k+1} \rightarrow p_{\alpha}, u^{k+1} \rightarrow u_{\alpha}$, and $v^{k+1} \rightarrow v_{\alpha}$ in (2.5)(2.7). Then, we suppose that the triples $\left(u_{\alpha}, v_{\alpha}, p_{\alpha}\right), \alpha=1,2$, satisfy (2.5)-(2.7). Testing (2.5) (written in terms of $\alpha=1$ ) with $u_{2}$ and testing (2.6) (written in terms of $\alpha=1$ ) with $v_{2}$ we have

$$
\begin{aligned}
-\gamma h^{2}\left[\phi^{\mathrm{ew}} D_{x} \mu_{1} \| u_{2}\right]_{\mathrm{ew}}= & h^{2}\left[D_{x} p_{1} \| u_{2}\right]_{\mathrm{ew}}+h^{2}\left[\eta^{\mathrm{ew}} u_{1} \| u_{2}\right]_{\mathrm{ew}}-2 h^{2}\left[D_{x}\left(v^{\mathrm{cc}} d_{x} u_{1}\right) \| u_{2}\right]_{\mathrm{ew}} \\
& -h^{2}\left[d_{y}\left\{v^{\mathrm{vc}}\left(D_{y} u_{1}+D_{x} v_{1}\right)\right\} \| u_{2}\right]_{\mathrm{ew}} \\
-\gamma h^{2}\left[\phi^{\mathrm{ns}} D_{y} \mu_{1} \| v_{2}\right]_{\mathrm{ns}}= & h^{2}\left[D_{y} p_{1} \| v_{2}\right]_{\mathrm{ns}}+h^{2}\left[\eta^{\mathrm{ns}} v_{1} \| v_{2}\right]_{\mathrm{ns}}-2 h^{2}\left[D_{y}\left(v^{\mathrm{cc}} d_{y} v_{1}\right) \| v_{2}\right]_{\mathrm{ns}} \\
& -h^{2}\left[d_{x}\left\{v^{\mathrm{vc}}\left(D_{x} v_{1}+D_{y} u_{1}\right)\right\} \| v_{2}\right]_{\mathrm{ns}} .
\end{aligned}
$$


Using the various summation-by-parts formulae from Appendix A and dropping boundary terms we have

$$
\begin{aligned}
\gamma h^{2}\left(\mu_{1} \| d_{x}\left(\phi^{\mathrm{ew}} u_{2}\right)\right)= & -h^{2}\left(p_{1} \| d_{x} u_{2}\right)+h^{2}\left[\eta^{\mathrm{ew}} u_{1} \| u_{2}\right]_{\mathrm{ew}}+2 h^{2}\left(v^{\mathrm{cc}} d_{x} u_{1} \| d_{x} u_{2}\right) \\
& +h^{2}\left\langle v^{\mathrm{vc}}\left(D_{y} u_{1}+D_{x} v_{1}\right) \| D_{y} u_{2}\right\rangle, \\
\gamma h^{2}\left(\mu_{1} \| d_{y}\left(\phi^{\mathrm{ns}} v_{2}\right)\right)= & -h^{2}\left(p_{1} \| d_{y} v_{2}\right)+h^{2}\left[\eta^{\mathrm{ns}} v_{1} \| v_{2}\right]_{\mathrm{ns}}+2 h^{2}\left(v^{\mathrm{cc}} d_{y} v_{1} \| d_{y} v_{2}\right) \\
& +h^{2}\left\langle v^{\mathrm{vc}}\left(D_{x} v_{1}+D_{y} u_{1}\right) \| D_{x} v_{2}\right\rangle .
\end{aligned}
$$

Adding the last two equations, and assuming the divergence-free condition (2.7) holds for $u_{2}, v_{2}$, we find

$$
\begin{aligned}
& \gamma h^{2}\left(\mu_{1} \| d_{x}\left(\phi^{\mathrm{ew}} u_{2}\right)+d_{y}\left(\phi^{\mathrm{ns}} v_{2}\right)\right) \\
= & h^{2}\left[\eta^{\mathrm{ew}} u_{1} \| u_{2}\right]_{\mathrm{ew}}+h^{2}\left[\eta^{\mathrm{ns}} v_{1} \| v_{2}\right]_{\mathrm{ns}}+2 h^{2}\left(v^{\mathrm{cc}} d_{x} u_{1} \| d_{x} u_{2}\right)+2 h^{2}\left(v^{\mathrm{cc}} d_{y} v_{1} \| d_{y} v_{2}\right) \\
& +h^{2}\left\langle v^{\mathrm{vc}}\left(D_{y} u_{1}+D_{x} v_{1}\right) \| D_{x} v_{2}+D_{y} u_{2}\right\rangle .
\end{aligned}
$$

\subsection{Unconditional unique solvability}

We first establish a lemma that will help prove solvability for (2.3)-(2.7). Let us define

$$
\begin{aligned}
& \stackrel{\ominus}{\bar{m} \times \bar{n}}:=\left\{\phi \in \mathcal{C}_{\bar{m} \times \bar{n}} \mid \mathbf{n} \cdot \nabla_{h} \phi=0 \text { and }(\phi \| \mathbf{1})=0\right\}, \\
& \dot{\mathcal{C}}_{m \times n}:=\left\{\phi \in \mathcal{C}_{m \times n} \mid(\phi \| \mathbf{1})=0\right\} .
\end{aligned}
$$

Note that $\operatorname{dim}\left(\mathcal{C}_{\bar{m} \times \bar{n}}\right)=\operatorname{dim}\left(\mathcal{C}_{m \times n}\right)$; in fact these spaces can be identified in a natural way. We also define the linear operator $\mathcal{L}\left(\mu_{\alpha}\right): \mathcal{C}_{\bar{m} \times \bar{n}} \rightarrow \mathcal{C}_{m \times n}$ via

$$
\mathcal{L}\left(\mu_{\alpha}\right):=-s \epsilon\left\{d_{x}\left(M^{\mathrm{ew}} D_{x} \mu_{\alpha}\right)+d_{y}\left(M^{\mathrm{ns}} D_{y} \mu_{\alpha}\right)\right\}+s\left\{d_{x}\left(\phi^{\mathrm{ew}} u_{\alpha}\right)+d_{y}\left(\phi^{\mathrm{ns}} v_{\alpha}\right)\right\},
$$

where, for a given grid function $\mu_{\alpha}$, the (staggered) grid functions $u_{\alpha} \in \mathcal{E}_{m \times \bar{n}}^{\mathrm{ew}}, v_{\alpha} \in \mathcal{E}_{\bar{m} \times n}^{\mathrm{ns}}$, and $p_{\alpha} \in \mathcal{C}_{m \times n}$ are the unique solutions to the discrete Brinkman equations

$$
\begin{aligned}
& -\gamma \phi^{\mathrm{ew}} D_{x} \mu_{\alpha}=D_{x} p_{\alpha}+\eta^{\mathrm{ew}} u_{\alpha}-2 D_{x}\left(v^{\mathrm{cc}} d_{x} u_{\alpha}\right)-d_{y}\left\{v^{\mathrm{vc}}\left(D_{y} u_{\alpha}+D_{x} v_{\alpha}\right)\right\} \\
& -\gamma \phi^{\mathrm{ns}} D_{y} \mu_{\alpha}=D_{y} p_{\alpha}+\eta^{\mathrm{ns}} v_{\alpha}-2 D_{y}\left(v^{\mathrm{cc}} d_{y} v_{\alpha}\right)-d_{x}\left\{v^{\mathrm{vc}}\left(D_{x} v_{\alpha}+D_{y} u_{\alpha}\right)\right\} \\
& d_{x} u_{\alpha}+d_{y} v_{\alpha}=0
\end{aligned}
$$

with homogeneous Dirichlet boundary conditions imposed on $u_{\alpha}$ and $v_{\alpha}$.

Lemma 2.1. Let $\phi^{\mathrm{ew}} \in \mathcal{E}_{m \times n}^{\mathrm{ew}}, \phi^{\mathrm{ns}} \in \mathcal{E}_{m \times n}^{\mathrm{ns}}$ be given. Then, for any $\phi \in \mathcal{C}_{m \times n}$, there exists a unique $\mu \in \mathcal{C}_{\bar{m} \times \bar{n}}$ that solves $\mathcal{L}(\mu)=\phi$. 
Proof. The unique existence of the staggered grid variables $u_{\alpha}, v_{\alpha}$, and $p_{\alpha}$ (for any $\mu_{\alpha}$ ) is a classical result for the simple Stokes case, i.e., $v \equiv 1, \eta \equiv 0$. The arguments can be straightforwardly extended for the discrete Brinkman equations so we omit the details here. Hence $\mathcal{L}$ is well-defined. Assuming that $\mu$ is a solution of $\mathcal{L}(\mu)=\phi$, the necessity of $(\phi \| \mathbf{1})=0$ follows from a simple summation-by-parts calculation utilizing the homogeneous boundary conditions. We omit this calculation as well.

To prove the lemma, it suffices to show that $\mathcal{L}$ is SPD restricted to $\mathcal{C}_{\bar{m} \times \bar{n}}$. To show symmetry we test (2.21) (written in terms of $\alpha=2$ ) with $\mu_{1}$ and sum by parts to obtain

$$
\begin{aligned}
h^{2}\left(\mathcal{L}\left(\mu_{2}\right) \| \mu_{1}\right)= & \operatorname{seh}^{2}\left\{\left[M^{\mathrm{ew}} D_{x} \mu_{2} \| D_{x} \mu_{1}\right]_{\mathrm{ew}}+\left[M^{\mathrm{ns}} D_{y} \mu_{2} \| D_{y} \mu_{1}\right]_{\mathrm{ns}}\right\} \\
& +\operatorname{sh}^{2}\left(d_{x}\left(\phi^{\mathrm{ew}} u_{2}\right)+d_{y}\left(\phi^{\mathrm{ns}} v_{2}\right) \| \mu_{1}\right) .
\end{aligned}
$$

Combining this last result with the calculation (2.18) we have

$$
\begin{aligned}
h^{2}\left(\mathcal{L}\left(\mu_{2}\right) \| \mu_{1}\right)= & s \in h^{2}\left\{\left[M^{\mathrm{ew}} D_{x} \mu_{2} \| D_{x} \mu_{1}\right]_{\mathrm{ew}}+\left[M^{\mathrm{ns}} D_{y} \mu_{2} \| D_{y} \mu_{1}\right]_{\mathrm{ns}}\right\} \\
& +\frac{h^{2}}{\gamma}\left\{\left[\eta^{\mathrm{ew}} u_{1} \| u_{2}\right]_{\mathrm{ew}}+\left[\eta^{\mathrm{ns}} v_{1} \| v_{2}\right]_{\mathrm{ns}}\right\} \\
& +\frac{2 h^{2}}{\gamma}\left\{\left(v^{\mathrm{cc}} d_{x} u_{1} \| d_{x} u_{2}\right)+\left(v^{\mathrm{cc}} d_{y} v_{1} \| d_{y} v_{2}\right)\right\} \\
& +\frac{h^{2}}{\gamma}\left\langle v^{\mathrm{vc}}\left(D_{y} u_{1}+D_{x} v_{1}\right) \| D_{x} v_{2}+D_{y} u_{2}\right\rangle .
\end{aligned}
$$

Thus $h^{2}\left(\mathcal{L}\left(\mu_{2}\right) \| \mu_{1}\right)=h^{2}\left(\mathcal{L}\left(\mu_{1}\right) \| \mu_{2}\right)$ and the operator is symmetric. Setting $\mu_{1}=\mu_{2}=\mu$ in the last result, we have

$$
\begin{aligned}
h^{2}(\mu \| \mathcal{L}(\mu))= & s h^{2}\left\{\left[M^{\mathrm{ew}} D_{x} \mu \| D_{x} \mu\right]_{\mathrm{ew}}+\left[M^{\mathrm{ns}} D_{y} \mu \| D_{y} \mu\right]_{\mathrm{ns}}\right\} \\
& +\frac{s h^{2}}{\gamma}\left\{\left[\eta^{\mathrm{ew}} u \| u\right]_{\mathrm{ew}}+\left[\eta^{\mathrm{ns}} v \| v\right]_{\mathrm{ns}}\right\} \\
& +\frac{2 s h^{2}}{\gamma}\left(v^{\mathrm{cc}} d_{x} u \| d_{x} u\right)+\frac{2 s h^{2}}{\gamma}\left(v^{\mathrm{cc}} d_{y} v \| d_{y} v\right) \\
& +\frac{s h^{2}}{\gamma}\left\langle v^{\mathrm{vc}}\left(D_{y} u+D_{x} v\right) \| D_{x} v+D_{y} u\right\rangle \\
\geq & s \in M_{0}\left\|\nabla_{h} \mu\right\|_{2}^{2} \\
\geq & 0,
\end{aligned}
$$

from which we conclude that $\mathcal{L}$ is positive semi-definite. Now $(\mu \| \mathcal{L}(\mu))=0$ only when $D_{x} \mu=0$ and $D_{y} \mu=0$ at every edge-centered point. But, the only way that this can happen is if $\mu$ is a constant function. With the restriction that $\mu$ is a mean zero function, we conclude that $\mu \equiv 0$. Hence $(\mu \| \mathcal{L}(\mu))=0$ only when $\mu \equiv 0$, which implies that $\mathcal{L}$ is SPD. 
We equip the spaces $\stackrel{\bullet}{\mathcal{C}}_{m \times n}$ and $\dot{\mathcal{C}}_{\bar{m}} \times \bar{n}$ with the inner product

$$
\left(\phi_{1} \| \phi_{2}\right)_{\mathcal{L}^{-1}}:=\left(\phi_{1} \| \mathcal{L}^{-1}\left(\phi_{2}\right)\right)=\left(\mathcal{L}^{-1}\left(\phi_{1}\right) \| \phi_{2}\right),
$$

for any $\phi_{1}, \phi_{2} \in \mathcal{C}_{m \times n} \cup \mathcal{C}_{\bar{m} \times \bar{n}}$.

Theorem 2.1 (unique solvability). The scheme (2.3)-(2.7) is uniquely solvable for any time step-size $s>0$ and is discretely mass conserving, i.e., $\left(\phi^{k+1}-\phi^{k} \| \mathbf{1}\right)=0$.

Proof. The proof is very similar to that of [32, Theorem 2]. In particular, one can show that the scheme (2.3)-(2.7) is equivalent to $\delta_{\phi} G\left(\phi^{k+1}\right)=0$, where $\delta_{\phi}$ is the discrete variation with respect to the grid variable $\phi$, i.e., the gradient. $G(\phi)$ is the strictly convex, coercive functional

$$
\begin{aligned}
G(\phi) & :=\frac{h^{2}}{2}\left(\phi-\phi^{k} \| \phi-\phi^{k}\right)_{\mathcal{L}^{-1}}+F_{c}(\phi)-h^{2}\left(\phi \| \delta_{\phi} F_{e}\left(\phi^{k}\right)\right) \\
& =\frac{h^{2}}{2}\left(\mathcal{L}^{-1}\left(\phi-\phi^{k}\right) \| \phi-\phi^{k}\right)+F_{c}(\phi)-h^{2}\left(\phi \| \delta_{\phi} F_{e}\left(\phi^{k}\right)\right)
\end{aligned}
$$

defined over the set (hyperplane) of admissible functions

$$
\mathcal{A}=\left\{\phi \in \mathcal{C}_{\bar{m} \times \bar{n}} \mid(\phi \| \mathbf{1})=\left(\phi^{k} \| \mathbf{1}\right)\right\} .
$$

We omit the details.

\subsection{Unconditional stability}

Theorem 2.2 (energy stability). The scheme (2.3)-(2.5) is unconditionally (strongly) energy stable, meaning that for any time step-size $s>0$,

$$
F\left(\phi^{k+1}\right) \leq F\left(\phi^{k}\right) .
$$

Moreover, for any positive integer $N$, we have

$$
F\left(\phi^{N}\right)+s \epsilon M_{0} \sum_{k=1}^{N}\left\|\nabla_{h} \mu^{k}\right\|_{2}^{2}+\frac{s \eta_{0}}{\gamma} \sum_{k=1}^{N}\left\|\mathbf{u}^{k}\right\|_{2}^{2}+\frac{s v_{0}}{2 \gamma} \sum_{k=1}^{N}\left\|\mathrm{D}_{h}\left(\mathbf{u}^{k}\right)\right\|_{2}^{2} \leq F\left(\phi^{0}\right),
$$

where

$$
\left\|\mathbf{u}^{k}\right\|_{2}^{2}:=h^{2}\left[u^{k} \| u^{k}\right]_{\mathrm{ew}}+h^{2}\left[v^{k} \| v^{k}\right]_{\mathrm{ns}}
$$

and

$$
\begin{aligned}
\frac{1}{2}\left\|\mathrm{D}_{h}\left(\mathbf{u}^{k}\right)\right\|_{2}^{2}:= & 2 h^{2}\left(d_{x} u^{k} \| d_{x} u^{k}\right)+2 h^{2}\left(d_{y} v^{k} \| d_{y} v^{k}\right) \\
& +h^{2}\left\langle D_{y} u^{k}+D_{x} v^{k} \| D_{x} v^{k}+D_{y} u^{k}\right\rangle .
\end{aligned}
$$


Proof. Testing Eq. (2.3) with $\mu^{k+1}$ and using (2.21) we have

$$
h^{2}\left(\phi^{k+1}-\phi^{k} \| \mu^{k+1}\right)=-h^{2}\left(\mathcal{L}\left(\mu^{k+1}\right) \| \mu^{k+1}\right) .
$$

Testing Eq. (2.4) with $\phi^{k+1}-\phi^{k}$, using (2.1), summing-by-parts, and dropping boundary terms we find

$$
\begin{aligned}
h^{2}\left(\phi^{k+1}-\phi^{k} \| \mu^{k+1}\right)= & F\left(\phi^{k+1}\right)-F\left(\phi^{k}\right) \\
& +\frac{1}{4 \epsilon}\left\|\left(\phi^{k+1}\right)^{2}-\left(\phi^{k}\right)^{2}\right\|_{2}^{2}+\frac{1}{2 \epsilon}\left\|\phi^{k+1}\left(\phi^{k+1}-\phi^{k}\right)\right\|_{2}^{2} \\
& +\frac{1}{2 \epsilon}\left\|\phi^{k+1}-\phi^{k}\right\|_{2}^{2}+\frac{\epsilon}{2}\left\|\nabla_{h} \phi^{k+1}-\nabla_{h} \phi^{k}\right\|_{2}^{2} .
\end{aligned}
$$

Subtracting Eq. (2.36) from (2.35) and rearranging terms we have

$$
\begin{aligned}
F\left(\phi^{k+1}\right)-F\left(\phi^{k}\right)= & -h^{2}\left(\mathcal{L}\left(\mu^{k+1}\right) \| \mu^{k+1}\right) \\
& -\frac{1}{4 \epsilon}\left\|\left(\phi^{k+1}\right)^{2}-\left(\phi^{k}\right)^{2}\right\|_{2}^{2}-\frac{1}{2 \epsilon}\left\|\phi^{k+1}\left(\phi^{k+1}-\phi^{k}\right)\right\|_{2}^{2} \\
& -\frac{1}{2 \epsilon}\left\|\phi^{k+1}-\phi^{k}\right\|_{2}^{2}-\frac{\epsilon}{2}\left\|\nabla_{h} \phi^{k+1}-\nabla_{h} \phi^{k}\right\|_{2}^{2} .
\end{aligned}
$$

Now, according to (2.27), we find

$$
\begin{aligned}
h^{2}\left(\mu^{k+1} \| \mathcal{L}\left(\mu^{k+1}\right)\right)= & s \in h^{2}\left[M^{\mathrm{ew}} D_{x} \mu^{k+1} \| D_{x} \mu^{k+1}\right]_{\mathrm{ew}}+s \epsilon h^{2}\left[M^{\mathrm{ns}} D_{y} \mu^{k+1} \| D_{y} \mu^{k+1}\right]_{\mathrm{ns}} \\
& +\frac{s h^{2}}{\gamma}\left[\eta^{\mathrm{ew}} u^{k+1} \| u^{k+1}\right]_{\mathrm{ew}}+\frac{s h^{2}}{\gamma}\left[\eta^{\mathrm{ns}} v^{k+1} \| v^{k+1}\right]_{\mathrm{ns}} \\
& +\frac{2 s h^{2}}{\gamma}\left(v^{\mathrm{cc}} d_{x} u^{k+1} \| d_{x} u^{k+1}\right)+\frac{2 s h^{2}}{\gamma}\left(v^{\mathrm{cc}} d_{y} v^{k+1} \| d_{y} v^{k+1}\right) \\
& +\frac{s h^{2}}{\gamma}\left\langle v^{\mathrm{vc}}\left(D_{y} u^{k+1}+D_{x} v^{k+1}\right) \| D_{x} v^{k+1}+D_{y} u^{k+1}\right\rangle \\
\geq & s \in M_{0}\left\|\nabla_{h} \mu^{k+1}\right\|_{2}^{2}+\frac{s \eta_{0}}{\gamma}\left\|\mathbf{u}^{k+1}\right\|_{2}^{2}+\frac{s v_{0}}{2 \gamma}\left\|\mathrm{D}_{h}\left(\mathbf{u}^{k+1}\right)\right\|_{2}^{2}
\end{aligned}
$$

The result (2.32) now follows upon summing the above from $k=0$ to $N-1$.

The next three lemmas, proved in $[32,35]$, will be needed later. The discrete Sobolev norms $\|\cdot\|_{1,2}$ and $\|\cdot\|_{1,2}$ are defined in the appendix.

Lemma 2.2. Suppose that $\phi \in \mathcal{C}_{\bar{m} \times \bar{n}}$. Then

$$
F(\phi) \geq C_{0}\|\phi\|_{1,2}^{2}-\frac{3 L_{x} L_{y}}{4 \epsilon},
$$

where $C_{0}=\min \left(\epsilon^{-1}, \epsilon\right) / 2$. 
Lemma 2.3. Suppose that $\phi \in \mathcal{C}_{\bar{m} \times \bar{n}}$ and $\left.\mathbf{n} \cdot \nabla_{h} \phi\right|_{\partial \Omega}=0$. Then

$$
\|\phi\|_{6} \leq C_{2}\|\phi\|_{1,2}
$$

where $C_{2}$ depends upon $L_{x}$ and $L_{y}$.

Lemma 2.4. Suppose that $\phi \in \mathcal{C}_{\bar{m} \times \bar{n}}$ and $\left.\mathbf{n} \cdot \nabla_{h} \phi\right|_{\partial \Omega}=0$. Then,

$$
\|\phi\|_{\infty} \leq C_{3}\|\phi\|_{2,2}, \quad C_{3}:=2 \sqrt{\max \left\{\frac{1}{L_{x} L_{y}}, \frac{L_{x}}{L_{y}}, \frac{L_{y}}{L_{x}}, \frac{L_{x} L_{y}}{2}\right\}} .
$$

We prove below that the scheme is stable in various discrete norms.

Theorem 2.3. Let $\Phi(x, y)$ be a smooth function on $\bar{\Omega}$, where $\Omega=\left(0, L_{x}\right) \times\left(0, L_{y}\right)$, with homogeneous Neumann boundary conditions. Set $\phi_{i, j}^{0}:=\Phi\left(x_{i}, y_{j}\right)$. Suppose $E$ is the continuous energy (1.1) and $\phi_{i, j}^{k} \in \mathcal{C}_{\bar{m} \times \bar{n}}$ is the $k^{\text {th }}$ solution of the scheme (2.3)-(2.5). Then

$$
\left\|\phi^{k}\right\|_{1,2} \leq \sqrt{\frac{E(\Phi)+C_{4} L_{x} L_{y}}{C_{0}}}=: C_{5},
$$

where $C_{4}>0$ and does not depend on either s or $h$. More specifically, with $s \cdot N=T$, we have

$$
\begin{aligned}
& \|\phi\|_{\ell^{\infty}\left(0, T ; H_{h}^{1}\right)}:=\max _{1 \leq k \leq N}\left\|\phi^{k}\right\|_{1,2} \leq C_{5}, \\
& \left\|\nabla_{h} \mu\right\|_{\ell^{2}\left(0, T ; \ell^{2}\right)}:=\sqrt{s \sum_{k=1}^{N}\left\|\nabla_{h} \mu^{k}\right\|_{2}^{2}} \leq \sqrt{\frac{C_{0}}{\epsilon M_{0}}} C_{5}=: C_{6}, \\
& \|\mathbf{u}\|_{\ell^{2}\left(0, T ; \ell^{2}\right)}:=\sqrt{s \sum_{k=1}^{N}\left\|\mathbf{u}^{k}\right\|_{2}^{2}} \leq \sqrt{\frac{\gamma C_{0}}{\eta_{0}}} C_{5}=: C_{7}, \\
& \left\|\mathrm{D}_{h}(\mathbf{u})\right\|_{\ell^{2}\left(0, T ; \ell^{2}\right)}:=\sqrt{s \sum_{k=1}^{N}\left\|\mathrm{D}_{h}\left(\mathbf{u}^{k}\right)\right\|_{2}^{2}} \leq \sqrt{\frac{2 \gamma C_{0}}{v_{0}}} C_{5}=: C_{8}, \\
& \|\phi\|_{\ell^{2}\left(0, T ; H_{h}^{2}\right)}:=\sqrt{s \sum_{k=1}^{N}\left\|\phi^{k}\right\|_{2,2}^{2}} \leq C_{9},
\end{aligned}
$$

where $C_{9}>0$ depends upon $T$ and $\Phi$, but is independent of $s$ and $h$.

Proof. The techniques used to prove (2.43)-(2.46) are similar to those in the proof of [32, Theorem 5] and the details are skipped. To prove (2.47), we begin by testing Eq. (2.4) with 
$\Delta_{h} \phi^{k+1}$ and sum by parts.

$$
\begin{aligned}
\epsilon\left\|\Delta_{h} \phi^{k+1}\right\|_{2}^{2}= & h^{2}\left(\nabla_{h} \mu^{k+1} \| \nabla_{h} \phi^{k+1}\right)+\frac{h^{2}}{\epsilon}\left(\left(\phi^{k+1}\right)^{3} \| \Delta_{h} \phi^{k+1}\right)+\frac{h^{2}}{\epsilon}\left(\nabla_{h} \phi^{k} \| \nabla_{h} \phi^{k+1}\right) \\
\leq & \frac{1}{2}\left\|\nabla_{h} \mu^{k+1}\right\|_{2}^{2}+\frac{1}{2}\left\|\nabla_{h} \phi^{k+1}\right\|_{2}^{2}+\frac{1}{2 \epsilon}\left\|\phi^{k+1}\right\|_{6}^{6}+\frac{\epsilon}{2}\left\|\Delta_{h} \phi^{k+1}\right\| \\
& +\frac{1}{2}\left\|\nabla_{h} \phi^{k}\right\|_{2}^{2}+\frac{1}{2}\left\|\nabla_{h} \phi^{k+1}\right\|_{2}^{2} .
\end{aligned}
$$

Then using (2.40) and (2.43)

$$
\begin{aligned}
\frac{\epsilon}{2}\left\|\Delta_{h} \phi^{k+1}\right\|_{2}^{2} & \leq \frac{1}{2}\left\|\nabla_{h} \mu^{k+1}\right\|_{2}^{2}+\frac{1}{2 \epsilon}\left\|\phi^{k+1}\right\|_{6}^{6}+\frac{3}{2} C_{5}^{2} \leq \frac{1}{2}\left\|\nabla_{h} \mu^{k+1}\right\|_{2}^{2}+\frac{1}{2 \epsilon} C_{2}^{6}\left\|\phi^{k+1}\right\|_{1,2}^{6}+\frac{3}{2} C_{5}^{2} \\
& \leq \frac{1}{2}\left\|\nabla_{h} \mu^{k+1}\right\|_{2}^{2}+\frac{1}{2 \epsilon} C_{2}^{6} C_{5}^{6}+\frac{3}{2} C_{5}^{2}
\end{aligned}
$$

Summing and using (2.43) and (2.44) gives the result.

Remark 2.1. Combining this last result with Lemma 2.4 naturally leads to

$$
\|\phi\|_{\ell^{2}\left(0, T ; \ell^{\infty}\right)}:=\sqrt{s \sum_{k=1}^{N}\left\|\phi^{k}\right\|_{\infty}^{2}} \leq C_{10}
$$

where $C_{10}>0$ depends upon $T$ and $\Phi$, but is independent of $s$ and $h$.

Remark 2.2. All of the results from this section can be easily extended to the cases where periodic boundary conditions or a mixture of periodic and physical boundary conditions are assumed.

\section{Numerical convergence study}

In this section we discuss aspects of the practical numerical solution of the scheme (2.3)(2.7) by using the nonlinear FAS multigrid method given in Appendix B. We present some convergence tests and perform some sample computations. The first set of tests we perform gives evidence that the multigrid solver is robust and converges with nearly optimal complexity. In another test we provide evidence that suggests our convex-splitting scheme is convergent - in other words, numerical solutions converge to the PDE solutions - as $s$ and $h \rightarrow 0$.

\subsection{Convergence and complexity of the multigrid solver}

To demonstrate the convergence and near optimal complexity (with respect to the grid size $h$ ) of the solver we provide evidence that the multigrid convergence rate is nearly 
independent of $h$. (The reader is referred to Appendix B for a description of the multigrid algorithm.) To this end, five separate tests, which are similar to those carried out in [11,32] for Cahn-Hilliard [11] and Cahn-Hilliard-Hele-Shaw [32] multigrid solvers, are performed. For the tests we take the initial data

$$
\phi_{i, j}^{0}=\frac{\left[1-\cos \left(\frac{4 x_{i} \pi}{3.2}\right)\right] \cdot\left[1-\cos \left(\frac{2 y_{j} \pi}{3.2}\right)\right]}{2}-1,
$$

and we use the parameters $L_{x}=L_{y}=3.2$. We fix the temporal step size at $s=1.0 \times 10^{-3}$ and report results at the $20^{\text {th }}$ time step, i.e., at the final $T=2.0 \times 10^{-2}$. We vary the spatial step size from $h=3.2 / 32$ to $h=3.2 / 512$ as indicated in the test results. There is only one tunable parameter in our nonlinear multigrid solver, namely $\lambda$, the number of multigrid smoothing sweeps, as defined [32, Appendix A]. Based on our experience, as well as established wisdom [25], we expect that the optimal value of $\lambda$ should be about 5 . Our experiments confirm this.

The stopping tolerance for the multigrid solver is taken to be $\|\boldsymbol{R}(\boldsymbol{\phi})\|_{2, \star} \leq \tau=1.0 \times$ $10^{-8}$, where the norm and the residual $\boldsymbol{R}(\boldsymbol{\phi})$ are similar to those defined in [32, Appendix

Table 1: The number of multigrid iterations required to reduce the norm of the residual below the tolerance $\tau=1.0 \times 10^{-8}$. The iteration counts are made at the $20^{\text {th }}$ time step $(k=20)$ using the fixed time step size $s=1.0 \times 10^{-3}$. The parameters for the test are given in the text and in the tables. The initial data are given by Eq. (3.1). The precise residual values for Tests 1 and 2 are shown in Fig. 1. Using the multigrid smoothing parameter $\lambda=2$ we observe, for a variety of parameter sets, that the required number of iterations is nearly independent of $h$.

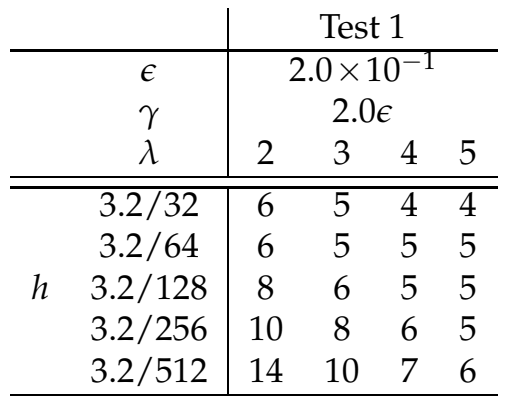

\begin{tabular}{c|cccc} 
& \multicolumn{4}{|c}{ Test 2 } \\
\hline$\epsilon$ & \multicolumn{4}{|c}{$1.0 \times 10^{-1}$} \\
$\gamma$ & \multicolumn{4}{c}{$2.0 \epsilon$} \\
$\lambda$ & 2 & 3 & 4 & 5 \\
\hline \hline $3.2 / 32$ & 5 & 4 & 4 & 4 \\
$3.2 / 64$ & 6 & 5 & 5 & 4 \\
$h .2 / 128$ & 7 & 6 & 5 & 5 \\
$3.2 / 256$ & 9 & 7 & 6 & 5 \\
$3.2 / 512$ & 12 & 9 & 7 & 6 \\
\hline
\end{tabular}

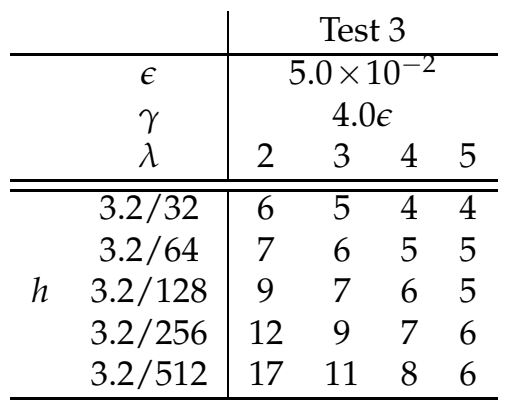

\begin{tabular}{cc|cccc} 
& \multicolumn{4}{|c}{ Test 4} \\
\hline$\epsilon$ & \multicolumn{4}{|c}{$5.0 \times 10^{-2}$} \\
$\gamma$ & 2 & $8.0 \epsilon$ & \\
$\lambda$ & 2 & 3 & 4 & 5 \\
\hline \hline $3.2 / 32$ & 6 & 5 & 5 & 4 \\
& $3.2 / 64$ & 7 & 6 & 5 & 5 \\
$h$ & $3.2 / 128$ & 10 & 8 & 6 & 6 \\
& $3.2 / 256$ & 13 & 9 & 7 & 6 \\
$3.2 / 512$ & 17 & 12 & 9 & 7 \\
\hline
\end{tabular}


A]. The number of multigrid iterations required to reduce the norm of the residual below the tolerance $\tau$ is given in Table 1 for various choices of $\epsilon, \gamma, \lambda$, and $h$. As can be seen in Table 1, using the multigrid smoothing parameter $\lambda=5$ we observe, for a variety of parameter choices, that the required number of iterations is nearly independent of $h$. The detailed residual values for Test 3 from Table 1 are given in graphical form in Fig. 1. For the case $\lambda=5$ note that the norm of the residual is reduced by approximately the same factor each time a multigrid iteration is executed, regardless of $h$. This is a typical feature of multigrid when it is operating with optimal complexity $[11,25]$. With $\lambda \leq 4$, we do not observe a similar feature. Significantly more multigrid iterations are required for smaller values of $h$.

\subsection{Convergence of the scheme as $s, h \rightarrow 0$}

Now we present the results of a test that suggest our scheme (2.3)-(2.7) is convergent as $s, h \rightarrow 0$. The initial data are given by Eq. (3.1) and the parameters are $L_{x}=L_{y}=3.2$, $\epsilon=2.0 \times 10^{-1}, \gamma=2.0 \epsilon$, and $T=4.0 \times 10^{-1}$, where $T$ is the final time. We expect that, at best, the global error in $\phi$ is $e_{t=T}=\mathcal{O}(s)+\mathcal{O}\left(h^{2}\right)$. Thus, if we choose a refinement path of the form $s=C h^{2}$, we have $e=\mathcal{O}\left(h^{2}\right)$, which is straightforward to confirm. We need only check that the global error is reduced by a factor of 4 when $h$ is reduced by a factor of 2 . In particular, we use the refinement path ${ }^{\dagger} s=0.4 h^{2}$. The full details of the convergence test are described in [10, Section 6.1], and for brevity we do not reproduce them here. The stopping tolerance for the solver is again taken to be $\tau=1.0 \times 10^{-8}$. The results of the test are reported in Table 2, and they give evidence that $e=\mathcal{O}\left(h^{2}\right)$ is indeed the case. In other words, a global error of the form $e=\mathcal{O}(s)+\mathcal{O}\left(h^{2}\right)$ is consistent with the test results. These results are comparable to those for the Cahn-Hilliard-Hele-Shaw system studied in [32].

Table 2: Errors and convergence rates of the convex-splitting scheme (2.3)-(2.5). Here the calculations are carried out for the variable $\phi$ only. Parameters are given in the text, and the initial data are defined in Eq. (3.1). The refinement path is $s=0.4 h^{2}$. Hence the results suggest global first-order convergence is attained, which was expected.

\begin{tabular}{||ccccc||}
\hline Grid sizes & $16^{2}-32^{2}$ & $32^{2}-64^{2}$ & $64^{2}-128^{2}$ & $128^{2}-256^{2}$ \\
\hline Error & $7.6239 \times 10^{-3}$ & $1.7582 \times 10^{-3}$ & $4.3425 \times 10^{-4}$ & $1.0833 \times 10^{-4}$ \\
\hline Rate & & 2.082 & 2.012 & 2.002 \\
\hline
\end{tabular}

\section{Applications}

In this section we apply the scheme presented in the last section to a couple of practical examples.

\footnotetext{
${ }^{\dagger}$ The reader should keep in mind that the choice of this refinement path has nothing to do with any time step restriction for stability or solvability. Recall that we can show that the scheme is unconditionally energy stable and unconditionally uniquely solvable.
} 

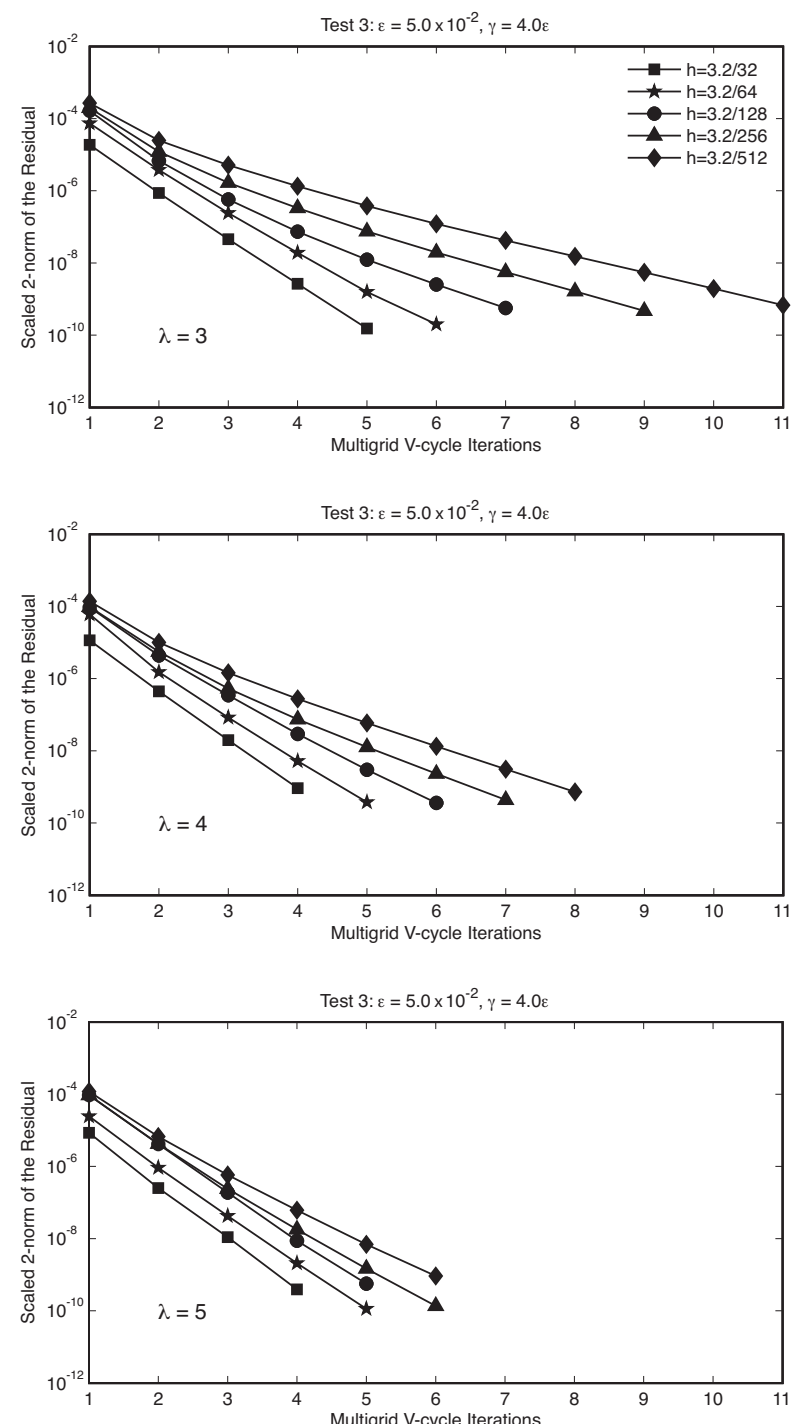

Figure 1: The reduction in the norm of the residual per $\mathrm{V}$-cycle iteration at time $t=2.0 \times 10^{-2}$ (20 full time steps with step size $\left.s=1.0 \times 10^{-3}\right)$. Parameters are given in the text and the initial data are given in Eq. (3.1). We show three cases, $\lambda=3,4$, and 5 for Test 3 represented in Table 1 . The results show a nearly $h$-independent reduction in the residual for the $\lambda=5$ case, though there is some deterioration in the convergence rate as $h \rightarrow 0$.

\subsection{Spinodal decomposition and energy dissipation}

Here we provide a couple of simulations of spinodal decomposition. We demonstrate energy dissipation at the numerical level and we show the effect of different values of the parameter $\gamma$. A similar test was given in [32] for the Cahn-Hilliard-Hele-Shaw equation, that is where the flow is given by Darcy's law. The results of the test are shown in Figs. 2 and 3 , and the parameters are $M(\phi)=\sqrt{(1+\phi)^{2}(1-\phi)^{2}+\epsilon^{2}}, s=0.005, h=6.4 / 256, \epsilon=0.03$, 

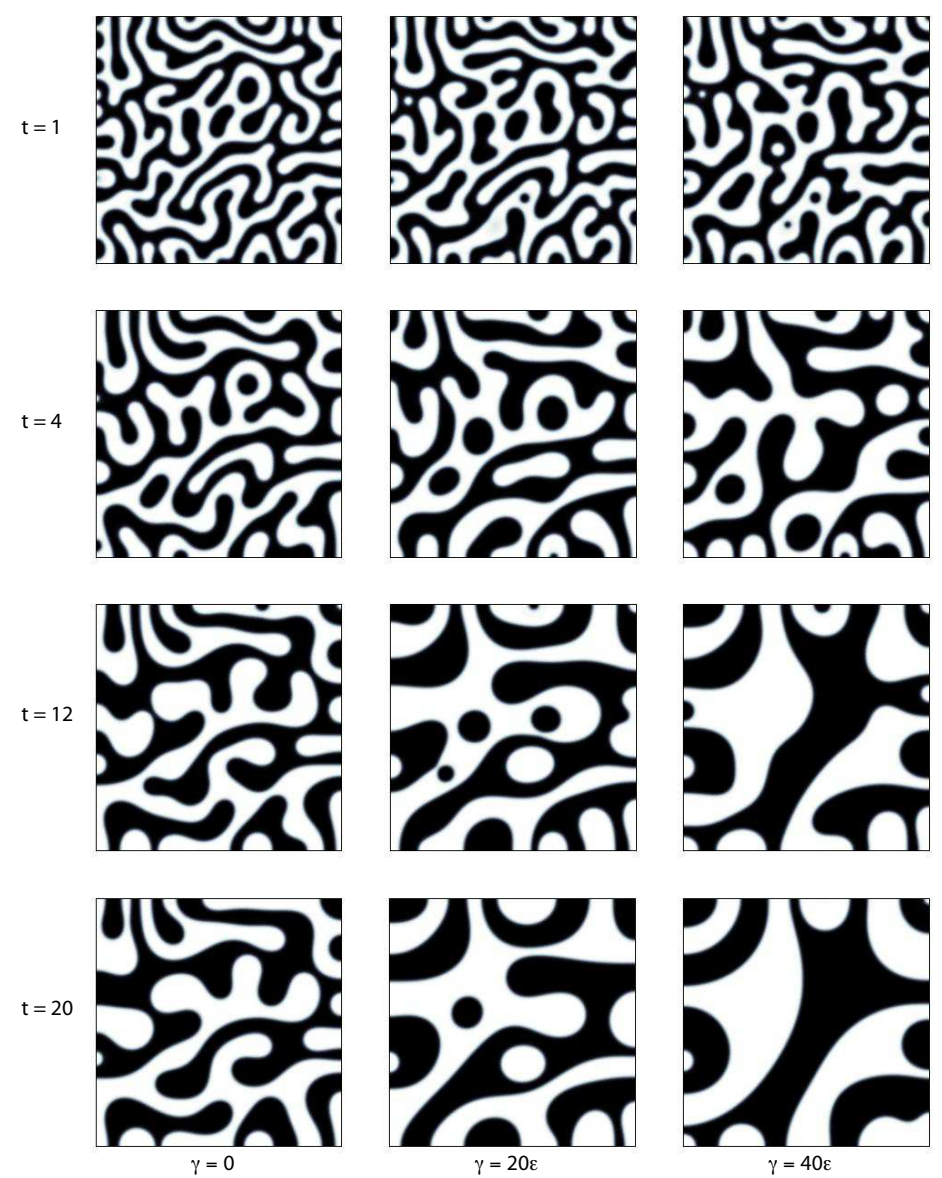

Figure 2: Phase separation and coarsening of a binary fluid with constant, uniform density. We show the filled contour plots of $\phi$ at various times. White means $\phi \approx 1$, black, $\phi \approx-1$. The parameters are $M(\phi)=$ $\sqrt{(1+\phi)^{2}(1-\phi)^{2}+\epsilon^{2}}, s=0.005, h=6.4 / 256, \epsilon=0.03, \gamma=4.0 \epsilon, \bar{\phi}=-0.05, \eta \equiv 0$, and $v \equiv 1$. The same initial data are used for all simulations. The chosen mobility limits the amount of diffusional coarsening, with respect to the case $M=1$, since $M=\epsilon \ll 1$ in the pure phases. The systems with larger excess surface tension, $\gamma$, tend to "straighten" their interfaces at a faster rate. This is reflected in the overall coarsening rates, as suggested in Fig.3.

$\gamma=4.0 \epsilon, \eta \equiv 0$, and $v \equiv 1$. The average composition is $\bar{\phi}=-0.05$. Initially, $\phi\left(x_{i}, y_{j}, 0\right)=\bar{\phi}+z_{i, j}$, where $z_{i, j} \in[-0.05,0.05]$ are random. After an initial rapid phase separation, the chosen mobility limits the amount of diffusional coarsening, with respect to the case $M=1$, since $M=\epsilon \ll 1$ in the pure phases. In this system coarsening is dominated by curve shortening via surface diffusion. See for example [4]. The systems with larger excess surface tension, $\gamma$, tend to "straighten" their interfaces at a faster rate. Coarsening rates are typically tied to the energy dissipation rates. Note that the correlation between higher values of $\gamma$ and higher coursing rates is reflected in Fig. 3 . We point out that, regardless of the parameter values of the space or time step sizes that the energy is always non-increasing in time. 


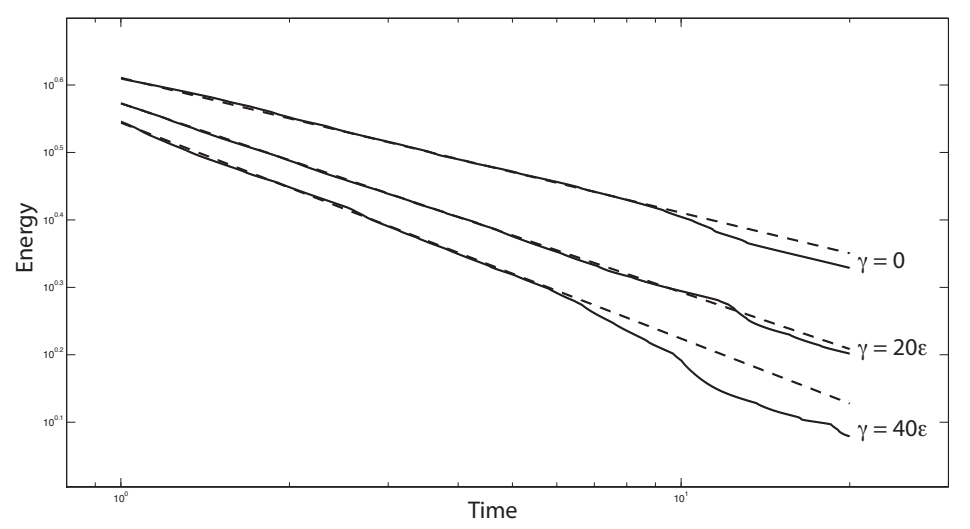

Figure 3: The computed discrete energies, $F$, plotted in log-log scale as functions of time (solid lines) for the three simulations in Fig. 2. The dashed lines are plots of the fit functions $4.08 t^{-0.20}(\gamma=0), 3.74 t^{-0.28}(\gamma=20 \epsilon)$, and $3.50 t^{-0.32}(\gamma=40 \epsilon), t \in[1,20]$ to avoid the phase separation regime. Clearly systems with higher excess surface tension $\gamma$ tend to coarsen faster. Note that wall effects become more influential at later times.

\subsection{Buoyancy-driven flows}

To simulate buoyancy-driven flow, we modify the Brinkman flow equation (1.3) as follows:

$$
-\nabla \cdot[v(\phi) \mathrm{D}(\mathbf{u})]+\eta(\phi) \mathbf{u}+\nabla p=-\gamma \phi \nabla \mu+\mathbf{b},
$$

where $\mathbf{b}$ is a buoyancy term that depends on the mass density. We assume that the mass density depends on $\phi$, i.e., $\rho=\rho(\phi)$, and we employ a Boussinesq type approximation:

$$
\mathbf{b}=-b(\phi) \hat{\mathbf{k}}, \quad b(\phi)=\chi\left(\phi-\phi_{0}\right),
$$

where $\phi_{0}$ is a constant (usually the average value of $\phi$ ), and $\chi$ is a constant. Hence, the corresponding scheme that we use for the buoyancy-driven flow is (2.3)-(2.7) with (2.6) replaced by

$$
\begin{aligned}
-\gamma A_{y} \phi^{k} D_{y} \mu^{k+1}-b\left(A_{y} \phi^{k}\right)= & D_{y} p^{k+1}+\eta\left(A_{y} \phi^{k}\right) v^{k+1}-2 D_{y}\left(v\left(\phi^{k}\right) d_{y} v^{k+1}\right) \\
& -d_{x}\left\{v\left(\mathcal{A} \phi^{k}\right)\left(D_{x} v^{k+1}+D_{y} u^{k+1}\right)\right\} .
\end{aligned}
$$

The simulation of the first buoyancy-driven flow is shown in Fig. 4. The numerical and physical parameters are $s=0.005, h=6.4 / 256, \epsilon=0.03, \gamma=4.0 \epsilon, \phi_{0}=\bar{\phi}=-0.05, \chi=10$, $\eta \equiv 0$, and $v \equiv 1$. We take the mobility to be

$$
M(\phi)=\sqrt{(1+\phi)^{4}(1-\phi)^{4}+\epsilon^{2}},
$$

so that $M=\epsilon$ in the pure phases, $\phi= \pm 1$. The mobility is $\sqrt{1+\epsilon^{2}} \approx 1$ on the level curve $\phi=0$. As before, a mobility of this type greatly limits the amount of diffusional coarsening in a system. Thus, most of the domain coarsening in these systems is driven by the 


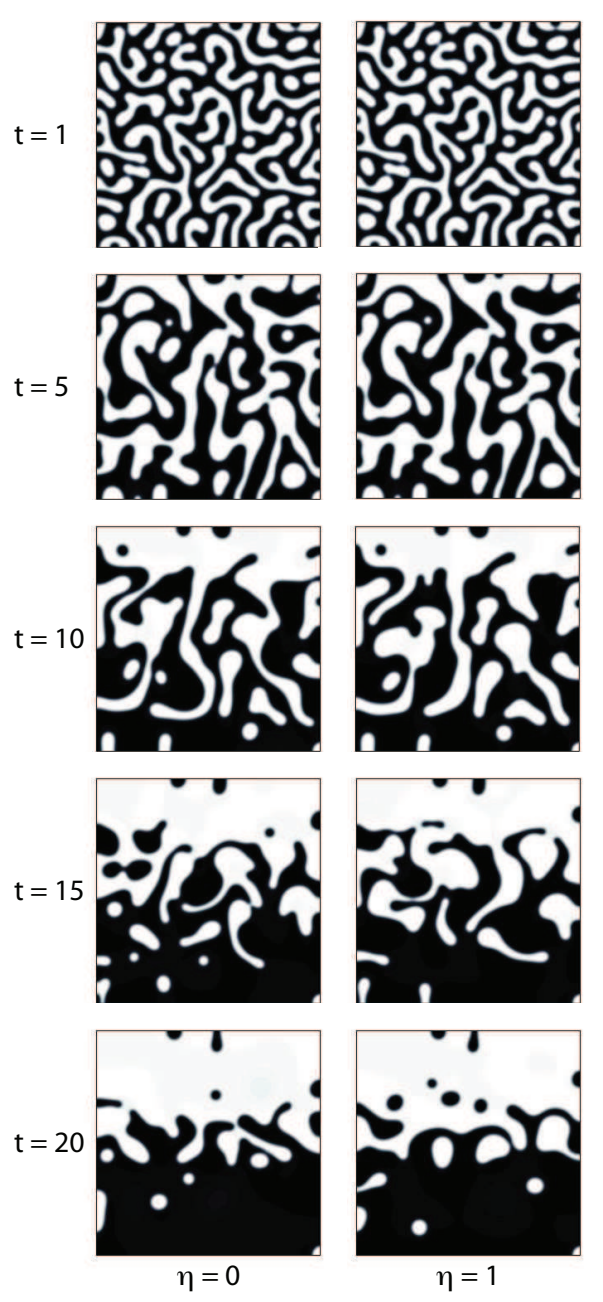

Figure 4: Phase separation of a binary fluid with variable density in a porous medium. Shown are filled contour plots of $\phi$ at various times. White means $\phi \approx 1$, black, $\phi \approx-1$. The parameters are $M(\phi)=$ $\sqrt{(1+\phi)^{4}(1-\phi)^{4}+\epsilon^{2}}, s=0.005, h=6.4 / 256, \epsilon=0.03, \gamma=4.0 \epsilon, \bar{\phi}=-0.05, b(\phi)=10(\phi-\bar{\phi})$, and $v \equiv 1$. Here the black fluid phase has greater mass density than the white. The left column shows the case $\eta \equiv 0$ (Stokes flow), the right column, the case $\eta \equiv 1$ (Brinkman flow). As before, the chosen mobility tends to limit the amount of diffusional coarsening.

fluid flow. The average composition is $\bar{\phi}=-0.05$, and initially, $\phi\left(x_{i}, y_{j}, 0\right)=\bar{\phi}+z_{i, j}$, where $z_{i, j} \in[-0.05,0.05]$ are random. Here the black fluid is heavier than the white fluid. After a very rapid phase separation process - which is similar to what was observed in the initial stages of the simulations in Fig. 2 - the black phase sinks and the white phase rises. The fluids mix in complicated ways as this process occurs. Note that the Stokes case $(\eta \equiv 0$, left column, Fig. 4) is qualitatively similar to the Brinkman case $(\eta \equiv 1$, right column, Fig. 4$)$. It does appear that individual particles in the Brinkman case have smaller shape factor - 
that is, they are rounder and more compact - than corresponding particles in the Stokes case.

As a second test of buoyancy-driven (Stokes) flow, we consider the case of a single bubble rising in a rectangular box. The parameters are $M(\phi)=0.1 \sqrt{(1+\phi)^{4}(1-\phi)^{4}+\epsilon^{2}}$, $s=0.005, h=2 / 256, \epsilon=0.01, \gamma=10.0 \epsilon, \phi_{0}=-0.05, b(\phi)=20\left(\phi-\phi_{0}\right), \eta \equiv 0$, and $v \equiv 1$. The results of the test are given in Fig. 5. As expected, the bubble rises, reaching an elliptical shape, and then deforms as it approaches the upper boundary.

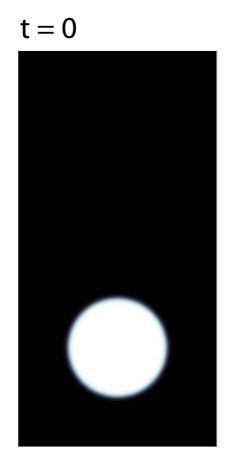

$$
t=30
$$

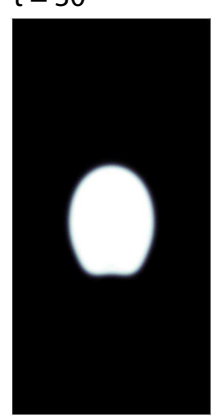

$$
t=60
$$

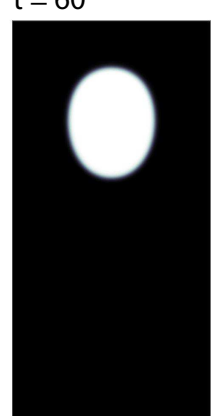

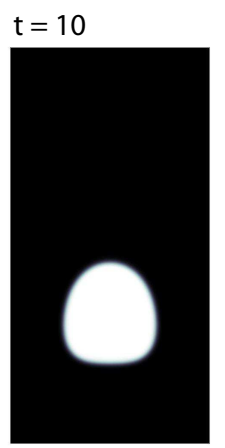

$\mathrm{t}=40$

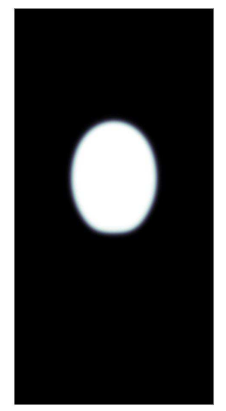

$t=70$

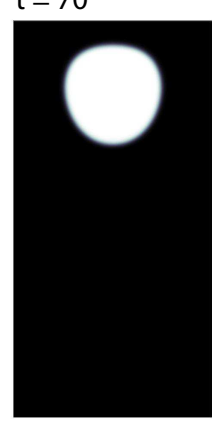

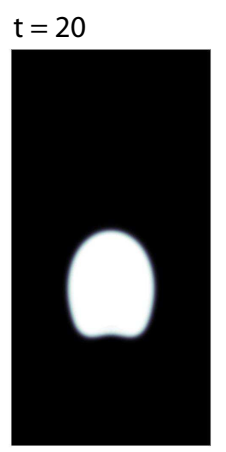

$\mathrm{t}=50$

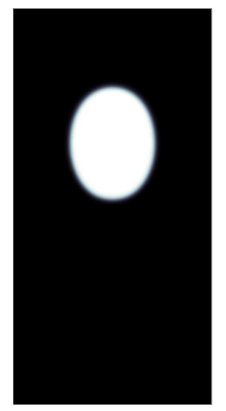

$\mathrm{t}=80$

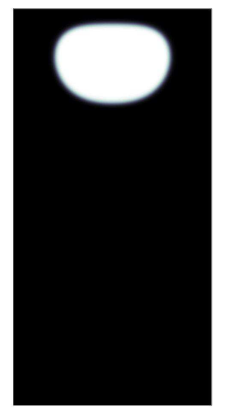

Figure 5: A rising bubble. Shown are filled contour plots of $\phi$ at various times. White means $\phi \approx 1$, black, $\phi \approx-1$. The parameters are $M(\phi)=0.1 \sqrt{(1+\phi)^{4}(1-\phi)^{4}+\epsilon^{2}}, s=0.005, h=2 / 256, \epsilon=0.01, \gamma=10.0 \epsilon, \bar{\phi}=-0.05$, $b(\phi)=20(\phi-\bar{\phi}), \eta \equiv 0$, and $v \equiv 1$. 


\subsection{Boundary-driven flows}

The last case we consider is that of boundary-driven flow. Specifically, we simulate the deformation of a particle in shear flow. The PDE model is given in Eqs. (1.2)-(1.4), however, we modify the boundary conditions as follows. Let $\mathbf{u}=(u, v)$ and $\Omega=\left(0, L_{x}\right) \times\left(0, L_{y}\right)$. We assume that all fields are periodic in the $x$ direction, and we set at $y=0$ and $y=L_{y}$, $\partial_{y} \phi=\partial_{y} \mu=0$, and $v=0$. For the first component of the velocity vector, $u=\sigma$ at $y=0$, and $u=-\sigma$ at $y=L_{y}$, where $\sigma>0$ is the shear rate. The scheme is the same as before. We only modify the numerical boundary condition (A.16) to accommodate the shear flow, and, of course, we modify all the boundary conditions, in a standard way, to allow for periodicity.

The results of two simple shear-flow simulations are reported in Figs. 6 and 7. In both tests, an initially round drop is suspend in the very center of box in which a shear flow is imposed via the boundary. The parameters are $M \equiv 1, s=0.001, h=6.4 / 256, L_{x}=12.8$, $L_{y}=6.4, \epsilon=0.03, \gamma=50.0 \epsilon, \eta \equiv 0$, and $v \equiv 1$. Here we only consider Stokes flow. The shear rates are $\sigma=2$ in Fig. 6 and $\sigma=1$ in Fig. 7 .
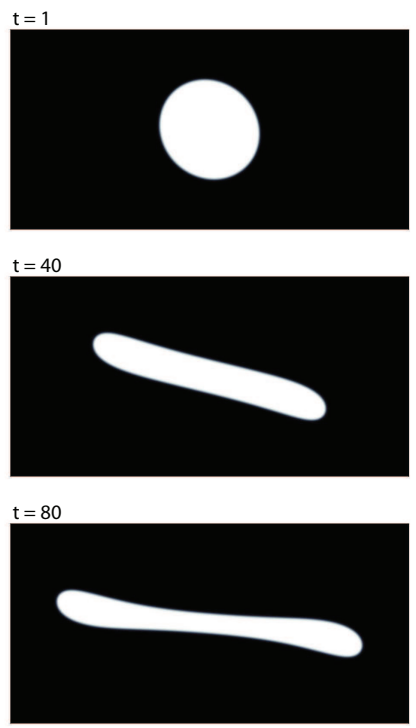
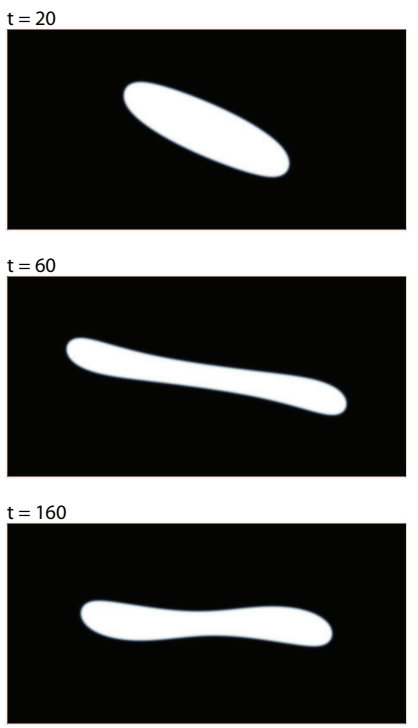

Figure 6: A suspended, initially round drop in shear flow. We show filled contour plots of $\phi$, where white indicates $\phi \approx 1$, black, $\phi \approx-1$. The parameters are $M \equiv 1, s=0.001, h=6.4 / 256, L_{x}=12.8, L_{y}=6.4, \epsilon=0.03$, $\gamma=50.0 \epsilon, \sigma=2, \eta \equiv 0$, and $v \equiv 1$. The right and left boundary conditions are taken to be periodic.

In Fig. 6, the shear rate is relatively modest, with respect to the surface tension. The particle is stretched, as expected, reaches a critical length, and then begins to retract. As it does, it rotates into the center line $y=L_{y} / 2$, where the flow velocity is zero. In Fig. 7 , where the shear rate is half that of the simulation in Fig. 6, a similar response of the particle to the flow is observed. Only here, as expected, the deformation of the drop is considerably less. 


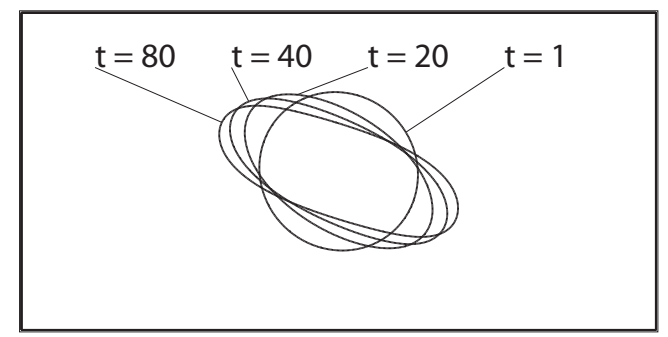

Figure 7: A suspended, initially round drop in shear flow. We show the $\phi=0$ level sets at four time snap shots. The parameters are $M \equiv 1, s=0.001, h=6.4 / 256, L_{x}=12.8, L_{y}=6.4, \epsilon=0.03, \gamma=50.0 \epsilon, \sigma=1, \eta \equiv 0$, and $v \equiv 1$. Note the shear rate is half that in Fig. 6 . The right and left boundary conditions are taken to be periodic.

\section{Concluding remarks}

In this paper we presented and analyzed a finite difference scheme for the Cahn-HilliardBrinkman system of equations. In particular, we proved that our scheme is uniquely solvable and energy stable, without any conditions on the time size $s$ or space step size $h$. The scheme is first-order accurate in time and second-order accurate in space. We presented a nonlinear multigrid method - based on a Vanka smoothing strategy for the Brinkman equation and a now standard multigrid strategy for the Cahn-Hilliard equation [32] - for solving the resulting algebraic equations. We showed that in some typical cases the convergence of our nonlinear multigrid solver can be near optimal with a suitable smoothing parameter. This is similar to the situation observed in [32] for the Cahn-Hilliard-Hele-Shaw equation.

This work builds upon our previous work on developing unconditionally energy stable schemes for the Cahn-Hilliard equation [22,23], the Cahn-Hilliard-Hele-Shaw equation $[9,32]$, and others $[10,28-30,35]$. The methods described herein can be immediately applied to the case where the Brinkman equation is replaced by a time-dependent Stokes/Brinkman equation of the form

$$
\partial_{t} \mathbf{u}-\nabla \cdot[v(\phi) \mathrm{D}(\mathbf{u})]+\eta(\phi) \mathbf{u}=-\nabla p-\gamma \phi \nabla \mu .
$$

We plan to extend our scheme for the full Cahn-Hilliard-Navier-Stokes equations, especially for the situation where the mass densities are so significantly different that the Boussinesq approximation is no longer valid [24].

While we have only considered a first-order scheme in time, it is also possible to construct second-order (in time) convex-splitting schemes. For example, a second-order scheme in strong form is as follows:

$$
\begin{aligned}
& \phi^{k+1}-\phi^{k}=s \nabla \cdot\left(\epsilon M\left(\tilde{\phi}^{k+\frac{1}{2}}\right) \nabla \mu^{k+\frac{1}{2}}-\tilde{\phi}^{k+\frac{1}{2}} \mathbf{u}^{k+\frac{1}{2}}\right), \\
& \mu^{k+\frac{1}{2}}=\frac{1}{2 \epsilon}\left[\left(\phi^{k+1}\right)^{2}+\left(\phi^{k}\right)^{2}\right] \phi^{k+\frac{1}{2}}-\frac{1}{\epsilon} \tilde{\phi}^{k+\frac{1}{2}}-\epsilon \Delta \phi^{k+\frac{1}{2}}, \\
& -\nabla \cdot\left(v\left(\tilde{\phi}^{k+\frac{1}{2}}\right) \mathrm{D}\left(\mathbf{u}^{k+\frac{1}{2}}\right)\right)+\eta\left(\tilde{\phi}^{k+\frac{1}{2}}\right) \mathbf{u}^{k+\frac{1}{2}}=-\nabla p^{k+\frac{1}{2}}-\gamma \tilde{\phi}^{k+\frac{1}{2}} \nabla \mu^{k+\frac{1}{2}},
\end{aligned}
$$


with $\nabla \cdot \mathbf{u}^{k+1 / 2}=0$, and where $\phi^{k+1 / 2}:=(1 / 2) \phi^{k+1}+(1 / 2) \phi^{k}, \tilde{\phi}^{k+1 / 2}:=(3 / 2) \phi^{k}-(1 / 2) \phi^{k-1}$, and $\phi^{-1}:=\phi^{0}$. This scheme is more difficult to analyze than the preliminary first-order scheme described herein. But, as suggested by our previous work $[10,21]$, we believe that all results established in this paper can be extended to this second-order scheme.

\section{Acknowledgments}

JS gratefully acknowledges support from the NSF grant DMS-095066 and AFOSR grant FA9550-11-1-0328, and SMW gratefully acknowledges support from the NSF through the grants DMS-1115390 and DMS-0818030 and funding through NIMBioS at the University of Tennessee.

\section{A Finite difference discretization on a staggered grid}

For simplicity, let us assume that $\Omega=\left(0, L_{x}\right) \times\left(0, L_{y}\right)$. Here we use the notation and results for cell-centered functions from $[32,35]$. The reader is directed to those references for more complete details. We begin with definitions of grid functions and difference operators needed for our discretization of two-dimensional space. Let $\Omega=\left(0, L_{x}\right) \times\left(0, L_{y}\right)$, with $L_{x}=m \cdot h$ and $L_{y}=n \cdot h$, where $m$ and $n$ are positive integers and $h>0$ is the spatial step size. Define $p_{r}:=(r-1 / 2) \cdot h$, where $r$ takes on integer and half-integer values. For any positive integer $\ell$, define $E_{\ell}=\left\{p_{r} \mid r=1 / 2, \cdots, \ell+1 / 2\right\}, C_{\ell}=\left\{p_{r} \mid r=1, \cdots, \ell\right\}$, $C_{\bar{\ell}}=\left\{p_{r} \cdot h \mid r=0, \cdots, \ell+1\right\}$. Define the function spaces

$$
\begin{array}{lll}
\mathcal{C}_{m \times n}=\left\{\phi: C_{m} \times C_{n} \rightarrow \mathbb{R}\right\}, & \mathcal{C}_{\bar{m} \times \bar{n}}=\left\{\phi: C_{\bar{m}} \times C_{\bar{n}} \rightarrow \mathbb{R}\right\}, \\
\mathcal{C}_{\bar{m} \times n}=\left\{\phi: C_{\bar{m}} \times C_{n} \rightarrow \mathbb{R}\right\}, & \mathcal{C}_{m \times \bar{n}}=\left\{\phi: C_{m} \times C_{\bar{n}} \rightarrow \mathbb{R}\right\}, \\
\mathcal{E}_{m \times n}^{\mathrm{ew}}=\left\{u: E_{m} \times C_{n} \rightarrow \mathbb{R}\right\}, & \mathcal{E}_{m \times n}^{\mathrm{ns}}=\left\{v: C_{m} \times E_{n} \rightarrow \mathbb{R}\right\}, \\
\mathcal{E}_{m \times \bar{n}}^{\mathrm{ew}}=\left\{u: E_{m} \times C_{\bar{n}} \rightarrow \mathbb{R}\right\}, & \mathcal{E}_{\bar{m} \times n}^{\mathrm{ns}}=\left\{v: C_{\bar{m}} \times E_{n} \rightarrow \mathbb{R}\right\}, \\
\mathcal{V}_{m \times n}=\left\{f: E_{m} \times E_{n} \rightarrow \mathbb{R}\right\} . & &
\end{array}
$$

We use the notation $\phi_{i, j}:=\phi\left(p_{i}, p_{j}\right)$ for cell-centered functions, those in the spaces $\mathcal{C}_{m \times n}$, $\mathcal{C}_{\bar{m} \times n}, \mathcal{C}_{m \times \bar{n}}$ or $\mathcal{C}_{\bar{m} \times \bar{n}}$. In component form east-west edge-centered functions, those in the spaces $\mathcal{E}_{m \times n}^{\mathrm{ew}}$ or $\mathcal{E}_{m \times \bar{n}}^{\mathrm{ew}}$, are identified via $u_{i+1 / 2, j}:=u\left(p_{i+1 / 2}, p_{j}\right)$. In component form northsouth edge-centered functions, those in the spaces $\mathcal{E}_{m \times n}^{\text {ns }}$, or $\mathcal{E}_{\bar{m} \times n^{\prime}}^{\mathrm{ns}}$, are identified via $u_{i+1 / 2, j}:=$ $u\left(p_{i+1 / 2}, p_{j}\right)$. The functions of $\mathcal{V}_{m \times n}$ are called vertex-centered functions. In component form vertex-centered functions are identified via $f_{i+1 / 2, j+1 / 2}:=f\left(p_{i+1 / 2}, p_{j+1 / 2}\right)$.

We will need the weighted $2 \mathrm{D}$ grid inner-products $(\cdot \| \cdot),[\cdot \| \cdot]_{\mathrm{ew}},[\cdot \| \cdot]_{\mathrm{ns}}$ that are defined in $[32,35]$. In addition to these, we will use the 2D grid inner product

$$
\begin{aligned}
\langle f \| g\rangle= & \frac{1}{4} \sum_{i=1}^{m} \sum_{j=1}^{n}\left(f_{i+\frac{1}{2}, j+\frac{1}{2}} g_{i+\frac{1}{2}, j+\frac{1}{2}}+f_{i+\frac{1}{2}, j-\frac{1}{2}} g_{i+\frac{1}{2}, j-\frac{1}{2}}\right. \\
& \left.+f_{i-\frac{1}{2}, j+\frac{1}{2}} g_{i-\frac{1}{2}, j+\frac{1}{2}}+f_{i-\frac{1}{2}, j-\frac{1}{2}} g_{i-\frac{1}{2}, j-\frac{1}{2}}\right), \quad f, g \in \mathcal{V}_{m \times n}^{\mathrm{ns}} .
\end{aligned}
$$


We will also need the following one-dimensional inner-products:

$$
\left(f_{\star, j+\frac{1}{2}} \mid g_{\star, j+\frac{1}{2}}\right)=\sum_{i=1}^{m} f_{i, j+\frac{1}{2}} g_{i, j+\frac{1}{2}}, \quad\left(f_{i+\frac{1}{2}, \star} \mid g_{i+\frac{1}{2}, \star}\right)=\sum_{j=1}^{n} f_{i+\frac{1}{2}, j} g_{i+\frac{1}{2}, j},
$$

where the first is defined for $f, g \in \mathcal{E}_{m \times n}^{\mathrm{ns}}$, and the second for $f, g \in \mathcal{E}_{m \times n}^{\mathrm{ew}}$; and

$$
\begin{aligned}
& {\left[u_{\star, j+\frac{1}{2}} \mid v_{\star, j+\frac{1}{2}}\right]=\frac{1}{2} \sum_{i=1}^{m}\left(u_{i-\frac{1}{2}, j+\frac{1}{2}} v_{i-\frac{1}{2}, j+\frac{1}{2}}+u_{i+\frac{1}{2}, j+\frac{1}{2}} v_{i+\frac{1}{2}, j+\frac{1}{2}}\right),} \\
& {\left[u_{i+\frac{1}{2}, \star} \mid v_{i+\frac{1}{2}, \star}\right]=\frac{1}{2} \sum_{j=1}^{n}\left(u_{i+\frac{1}{2}, j-\frac{1}{2}} v_{i+\frac{1}{2}, j-\frac{1}{2}}+u_{i+\frac{1}{2}, j+\frac{1}{2}} v_{i+\frac{1}{2}, j+\frac{1}{2}}\right) .}
\end{aligned}
$$

which are both defined for $u, v \in \mathcal{V}_{m \times n}$. See, for example, [35, Section 2.1].

The reader is referred to $[32,35]$ for the precise definitions of the edge-to-center difference operators $d_{x}: \mathcal{E}_{m \times n}^{\mathrm{ew}} \rightarrow \mathcal{C}_{m \times n}$ and $d_{y}: \mathcal{E}_{m \times n}^{\text {ns }} \rightarrow \mathcal{C}_{m \times n}$; the $x$-dimension center-to-edge average and difference operators, respectively, $A_{x}, D_{x}: \mathcal{C}_{\bar{m} \times n} \rightarrow \mathcal{E}_{m \times n}^{\mathrm{ew}} ;$ the $y$-dimension center-to-edge average and difference operators, respectively, $A_{y}, D_{y}: \mathcal{C}_{m \times \bar{n}} \rightarrow \mathcal{E}_{m \times n}^{\text {ns }}$; and the standard 2D discrete Laplacian, $\Delta_{h}: \mathcal{C}_{\bar{m} \times \bar{n}} \rightarrow \mathcal{C}_{m \times n}$. In addition to these, we also need some operations involving vertex-centered functions. The center-to-vertex average $\mathcal{A}: \mathcal{C}_{\bar{m} \times \bar{n}} \rightarrow \mathcal{V}_{m \times n}$ is defined component-wise as

$$
\mathcal{A} \phi_{i+\frac{1}{2}, j+\frac{1}{2}}=\frac{1}{4}\left(\phi_{i, j}+\phi_{i+1, j}+\phi_{i, j+1}+\phi_{i+1, j+1}\right), \quad i=0, \cdots, m+1, \quad j=0, \cdots, n+1 .
$$

The edge-to-vertex differences are defined as follows: $D_{x}: \mathcal{E}_{\bar{m} \times n}^{\mathrm{ew}} \rightarrow \mathcal{V}_{m \times n}$ is defined componentwise as

$$
D_{x} v_{i+\frac{1}{2}, j+\frac{1}{2}}=\frac{1}{h}\left(v_{i+1, j+\frac{1}{2}}-v_{i, j+\frac{1}{2}}\right), \quad i=0, \cdots, m+1, \quad j=0, \cdots, n,
$$

and $D_{y}: \mathcal{E}_{m \times \bar{n}}^{\text {ns }} \rightarrow \mathcal{V}_{m \times n}$ is defined component-wise as

$$
D_{y} u_{i+\frac{1}{2}, j+\frac{1}{2}}=\frac{1}{h}\left(v_{i+\frac{1}{2}, j+1}-v_{i+\frac{1}{2}, j}\right), \quad i=0, \cdots, m+1, \quad j=0, \cdots, n .
$$

In this paper we use grid functions satisfying homogeneous Neumann boundary conditions. Specifically, we shall say the cell-centered function $\phi \in \mathcal{C}_{\bar{m} \times \bar{n}}$ satisfies homogeneous Neumann boundary conditions if and only if

$$
\begin{array}{lll}
\phi_{m+1, j}=\phi_{m, j}, & \phi_{0, j}=\phi_{1, j}, & j=1, \cdots, n, \\
\phi_{i, n+1}=\phi_{i, n}, & \phi_{i, 0}=\phi_{i, 1}, & i=0, \cdots, m+1 .
\end{array}
$$

We use the notation $\mathbf{n} \cdot \nabla_{h} \phi=0$ to indicate that $\phi$ satisfies (A.13) and (A.14). Now, let $u \in \mathcal{E}_{m \times \bar{n}}^{\mathrm{ew}}$. We say that $u=0$ on the boundary if and only if

$$
\begin{array}{lll}
u_{m+\frac{1}{2}, j}=0, & u_{\frac{1}{2}, j}=0, & j=1, \cdots, n, \\
A_{y} u_{i+\frac{1}{2}, n+\frac{1}{2}}=0, & A_{y} u_{i+\frac{1}{2}, \frac{1}{2}}=0, & i=0, \cdots, m .
\end{array}
$$


Likewise, let $v \in \mathcal{E}_{\bar{m} \times n}^{\mathrm{ns}}$. We say that $v=0$ on the boundary if and only if

$$
\begin{array}{lll}
v_{i, n+\frac{1}{2},}=0, & v_{i, \frac{1}{2}}=0, & i=1, \cdots, m, \\
A_{x} v_{m+\frac{1}{2}, j+\frac{1}{2}}=0, & A_{x} v_{\frac{1}{2}, j+\frac{1}{2}}=0, & j=0, \cdots, n .
\end{array}
$$

We will also use combinations of homogeneous Dirichlet and periodic boundary conditions, or homogeneous Neumann and periodic boundary conditions in some instances, though we suppress the precise definitions.

We will use the grid function norms defined in [32,35]: for $\phi \in \mathcal{C}_{m \times n}, 1 \leq p<\infty$,

$$
\|\phi\|_{p}:=\left(h^{2} \sum_{i=1}^{m} \sum_{j=1}^{n}\left|\phi_{i, j}\right|^{p}\right)^{1 / p} \quad \text { and } \quad\|\phi\|_{\infty}=\max _{\substack{1 \leq i \leq m \\ 1 \leq j \leq n}}\left|\phi_{i, j}\right| .
$$

For any $\phi \in \mathcal{C}_{\bar{m} \times \bar{n}}$, we define

$$
\left\|\nabla_{h} \phi\right\|_{2}:=\sqrt{h^{2}\left[D_{x} \phi \| D_{x} \phi\right]_{\mathrm{ew}}+h^{2}\left[D_{y} \phi \| D_{y} \phi\right]_{\mathrm{ew}}},
$$

and we need the following discrete Sobolev norms: $\|\cdot\|_{0,2}:=\|\cdot\|_{2}$,

$$
\|\phi\|_{1,2}=\sqrt{\|\phi\|_{2}^{2}+\left\|\nabla_{h} \phi\right\|_{2}^{2}}, \quad\|\phi\|_{2,2}=\sqrt{\|\phi\|_{1,2}^{2}+\left\|\Delta_{h} \phi\right\|_{2}^{2}} .
$$

Using the definitions given in this appendix and in [32,35], we obtain the following summation-by-parts formulae:

Proposition A.1 (summation-by-parts). If $\phi \in \mathcal{C}_{\bar{m} \times n} \cup \mathcal{C}_{\bar{m} \times \bar{n}}$ and $f \in \mathcal{E}_{m \times n}^{\text {ew }}$ then

$$
h^{2}\left[D_{x} \phi \| f\right]_{\mathrm{ew}}=-h^{2}\left(\phi \| d_{x} f\right)-h\left(A_{x} \phi_{\frac{1}{2}, \star} \mid f_{\frac{1}{2}, \star}\right)+h\left(A_{x} \phi_{m+\frac{1}{2}, \star} \mid f_{m+\frac{1}{2}, \star}\right),
$$

and if $\phi \in \mathcal{C}_{m \times \bar{n}} \cup \mathcal{C}_{\bar{m} \times \bar{n}}$ and $f \in \mathcal{E}_{m \times n}^{\text {ns }}$ then

$$
h^{2}\left[D_{y} \phi \| f\right]_{\mathrm{ns}}=-h^{2}\left(\phi \| d_{y} f\right)-h\left(A_{y} \phi_{\star, \frac{1}{2}} \mid f_{\star, \frac{1}{2}}\right)+h\left(A_{y} \phi_{\star, n+\frac{1}{2}} \mid f_{\star, n+\frac{1}{2}}\right) .
$$

If $f \in \mathcal{V}_{m \times n}$ and $g \in \mathcal{E}_{\bar{m} \times n}^{\mathrm{ns}}$ then

$$
h^{2} \cdot\left[d_{x} f \| g\right]_{\mathrm{ns}}=-h^{2} \cdot\left\langle f \| D_{x} g\right\rangle-h \cdot\left[A_{x} g_{\frac{1}{2}, \star} \mid f_{\frac{1}{2}, \star}\right]+h \cdot\left[A_{x} g_{m+\frac{1}{2}, \star} \mid f_{m+\frac{1}{2}, \star}\right],
$$

and if $f \in \mathcal{V}_{m \times n}$ and $g \in \mathcal{E}_{m \times \bar{n}}^{\mathrm{ew}}$ then

$$
h^{2} \cdot\left[d_{y} f \| g\right]_{\mathrm{ew}}=-h^{2} \cdot\left\langle f \| D_{y} g\right\rangle-h \cdot\left[A_{x} g_{\star, \frac{1}{2}} \mid f_{\star, \frac{1}{2}}\right]+h \cdot\left[A_{x} g_{\star, n+\frac{1}{2}} \mid f_{\star, n+\frac{1}{2}}\right] .
$$


Proposition A.2 (discrete Green's first identity). Let $\phi, \psi \in \mathcal{C}_{\bar{m} \times \bar{n}}$. Then

$$
\begin{aligned}
& h^{2}\left[D_{x} \phi \| D_{x} \psi\right]_{\mathrm{ew}}+h^{2}\left[D_{y} \phi \| D_{y} \psi\right]_{\mathrm{ns}} \\
= & -h^{2}\left(\phi \| \Delta_{h} \psi\right)-h\left(A_{x} \phi_{\frac{1}{2}, \star} \mid D_{x} \psi_{\frac{1}{2}, \star}\right)+h\left(A_{x} \phi_{m+\frac{1}{2}, \star} \mid D_{x} \psi_{m+\frac{1}{2}, \star}\right) \\
& -h\left(A_{y} \phi_{\star, \frac{1}{2}} \mid D_{y} \psi_{\star, \frac{1}{2}}\right)+h\left(A_{y} \phi_{\star, n+\frac{1}{2}} \mid D_{y} \psi_{\star, n+\frac{1}{2}}\right) .
\end{aligned}
$$

Proposition A.3 (discrete Green's second identity). Let $\phi, \psi \in \mathcal{C}_{\bar{m} \times \bar{n}}$. Then

$$
\begin{aligned}
& h^{2}\left(\phi \| \Delta_{h} \psi\right) \\
= & h^{2}\left(\Delta_{h} \phi \| \psi\right)+h\left(A_{x} \phi_{m+\frac{1}{2}, \star} \mid D_{x} \psi_{m+\frac{1}{2}, \star}\right)-h\left(D_{x} \phi_{m+\frac{1}{2}, \star} \mid A_{x} \psi_{m+\frac{1}{2}, \star}\right) \\
& -h\left(A_{x} \phi_{\frac{1}{2}, \star} \mid D_{x} \psi_{\frac{1}{2}, \star}\right)+h\left(D_{x} \phi_{\frac{1}{2}, \star} \mid A_{x} \psi_{\frac{1}{2}, \star}\right)+h\left(A_{y} \phi_{\star, n+\frac{1}{2}} \mid D_{y} \psi_{\star, n+\frac{1}{2}}\right) \\
& -h\left(D_{y} \phi_{\star, n+\frac{1}{2}} \mid A_{y} \psi_{\star, n+\frac{1}{2}}\right)-h\left(A_{y} \phi_{\star, \frac{1}{2}} \mid D_{y} \psi_{\star, \frac{1}{2}}\right)+h\left(D_{y} \phi_{\star, \frac{1}{2}} \mid A_{y} \psi_{\star, \frac{1}{2}}\right) .
\end{aligned}
$$

We remark that these formulae have straightforward extensions to three dimensions.

\section{B Multigrid solver}

The nonlinear multigrid solver for our Cahn-Hilliard-Brinkman scheme (2.3)-(2.7) is similar in many respects to that described for the Cahn-Hilliard-Hele-Shaw problem in our previous paper [32, Appendix A]. The primary difference is that in the present case we decouple the smoothing operator. We first perform a relaxation on the Cahn-Hilliard equations (2.3) and (2.4); then we relax the flow equations (2.5)-(2.7) separately. Specifically, in the proposed smoother, for each grid cell $(i, j)$ we update the unknowns $\phi_{i, j}^{k+1}$ and $\mu_{i, j}^{k+1}$ first using a nonlinear Gauss-Seidel method, similar to what is described in [32, Appendix A]. Then we update the five variables $u_{i \pm 1 / 2, j^{\prime}}^{k+1}, v_{i, j \pm 1 / 2}^{k+1}$, and $p_{i, j}^{k+1}$ using a Vankatype smoothing strategy $[19,25,26,31]$ with the updated values for $\phi_{i, j}^{k+1}$ and $\mu_{i, j}^{k+1}$. Note that $u$ and $v$ are edge-centered variables and this contributes to the complication of the method.

Here we give the full details of the Vanka smoothing strategy and refer the reader to [32, Appendix A] for the remaining details of the solver. To simplify the discussion, we drop the time superscript $k+1$ on the unknowns $u, v, p$, and $\mu$ in (2.5)-(2.7). And, as before, we define the known grid functions

$$
v^{\mathrm{cc}}:=v\left(\phi^{k}\right), \quad v^{\mathrm{vc}}:=v\left(\mathcal{A} \phi^{k}\right), \quad \phi^{\mathrm{ew}}:=A_{x} \phi^{k}, \quad \phi^{\mathrm{ns}}:=A_{y} \phi^{k},
$$

and

$$
\eta^{\mathrm{ew}}:=\eta\left(\phi^{\mathrm{ew}}\right), \quad \eta^{\mathrm{ns}}:=\eta\left(\phi^{\mathrm{ns}}\right)
$$


where we have dropped the superscript $k$. We write the flow equations (2.5)-(2.7) in the form

$$
N^{u}=S^{u}, \quad N^{v}=S^{v}, \quad N^{p}=S^{p},
$$

where

$$
\begin{aligned}
& N^{u}:=-2 D_{x}\left(v^{\mathrm{cc}} d_{x} u\right)-d_{y}\left[v^{\mathrm{vc}}\left(D_{y} u+D_{x} v\right)\right]+\eta^{\mathrm{ew}} u+D_{x} p+\gamma \phi^{\mathrm{ew}} D_{x} \mu, \\
& N^{v}:=-2 D_{y}\left(v^{\mathrm{cc}} d_{y} v\right)-d_{x}\left[v^{\mathrm{vc}}\left(D_{x} v+D_{y} u\right)\right]+\eta^{\mathrm{ns}} v+D_{y} p+\gamma \phi^{\mathrm{ns}} D_{y} \mu, \\
& N^{p}:=-d_{x} u-d_{y} v,
\end{aligned}
$$

and, in this case, $S^{u}=S^{v}=S^{p}=0$. The discussion that follows simplifies significantly in the case of constant viscosity. For example, when $v \equiv 1$, we have

$$
\begin{aligned}
& N^{u}:=-D_{x}\left(d_{x} u\right)-d_{y}\left(D_{y} u\right)+\eta^{\mathrm{ew}} u+D_{x} p+\gamma \phi^{\mathrm{ew}} D_{x} \mu, \\
& N^{v}:=-D_{y}\left(d_{y} v\right)-d_{x}\left(D_{x} v\right)+\eta^{\mathrm{ns}} v+D_{y} p+\gamma \phi^{\mathrm{ns}} D_{y} \mu,
\end{aligned}
$$

upon using the divergence-free condition. We will not pursue this simplification here any further.

In component form, Eqs. (B.4)-(B.4) are written as

$$
\begin{aligned}
N_{i+\frac{1}{2}, j}^{u}= & -2 D_{x}\left(v^{\mathrm{cc}} d_{x} u\right)_{i+\frac{1}{2}, j}-d_{y}\left[v^{\mathrm{vc}}\left(D_{y} u+D_{x} v\right)\right]_{i+\frac{1}{2}, j}+\eta_{i+\frac{1}{2}, j}^{\mathrm{ew}} u_{i+\frac{1}{2}, j} \\
& +D_{x} p_{i+\frac{1}{2}, j}+\gamma \phi_{i+\frac{1}{2}, j}^{\mathrm{ew}} D_{x} \mu_{i+\frac{1}{2}, j} \\
= & -\frac{2}{h^{2}}\left[v_{i+1, j}^{\mathrm{cc}}\left(u_{i+\frac{3}{2}, j}-u_{i+\frac{1}{2}, j}\right)-v_{i, j}^{\mathrm{cc}}\left(u_{i+\frac{1}{2}, j}-u_{i-\frac{1}{2}, j}\right)\right] \\
& -\frac{1}{h^{2}}\left[v_{i+\frac{1}{2}, j+\frac{1}{2}}^{\mathrm{vc}}\left(u_{i+\frac{1}{2}, j+1}-u_{i+\frac{1}{2}, j}\right)-v_{i+\frac{1}{2}, j-\frac{1}{2}}^{\mathrm{vc}}\left(u_{i+\frac{1}{2}, j}-u_{i+\frac{1}{2}, j-1}\right)\right] \\
& -\frac{1}{h^{2}}\left[v_{i+\frac{1}{2}, j+\frac{1}{2}}^{\mathrm{vc}}\left(v_{i+1, j+\frac{1}{2}}-v_{i, j+\frac{1}{2}}\right)-v_{i+\frac{1}{2}, j-\frac{1}{2}}^{\mathrm{vc}}\left(v_{i+1, j-\frac{1}{2}}-v_{i, j-\frac{1}{2}}\right)\right] \\
& +\eta_{i+\frac{1}{2}, j}^{\mathrm{ew}} u_{i+\frac{1}{2}, j}+\frac{1}{h}\left(p_{i+1, j}-p_{i, j}\right)+\frac{\gamma}{h} \phi_{i+\frac{1}{2}, j}^{\mathrm{ew}}\left(\mu_{i+1, j}-\mu_{i, j}\right),
\end{aligned}
$$

and

$$
\begin{aligned}
N_{i, j+\frac{1}{2}}^{v}= & -2 D_{y}\left(v^{\mathrm{cc}} d_{y} v\right)_{i, j+\frac{1}{2}}-d_{x}\left[v^{\mathrm{vc}}\left(D_{x} v+D_{y} u\right)\right]_{i, j+\frac{1}{2}}+\eta_{i, j+\frac{1}{2}}^{\mathrm{ns}} v_{i, j+\frac{1}{2}} \\
& +D_{y} p_{i, j+\frac{1}{2}}+\gamma \phi_{i, j+\frac{1}{2}}^{\mathrm{ns}} D_{y} \mu_{i, j+\frac{1}{2}} \\
= & -\frac{2}{h^{2}}\left[v_{i, j+1}^{\mathrm{cc}}\left(v_{i, j+\frac{3}{2}}-v_{i, j+\frac{1}{2}}\right)-v_{i, j}^{\mathrm{cc}}\left(v_{i, j+\frac{1}{2}}-v_{i, j-\frac{1}{2}}\right)\right] \\
& -\frac{1}{h^{2}}\left[v_{i+\frac{1}{2}, j+\frac{1}{2}}^{\mathrm{vc}}\left(v_{i+1, j+\frac{1}{2}}-v_{i, j+\frac{1}{2}}\right)-v_{i-\frac{1}{2}, j+\frac{1}{2}}^{\mathrm{vc}}\left(v_{i, j+\frac{1}{2}}-v_{i-1, j+\frac{1}{2}}\right)\right] \\
& -\frac{1}{h^{2}}\left[v_{i+\frac{1}{2}, j+\frac{1}{2}}^{\mathrm{vc}}\left(u_{i+\frac{1}{2}, j+1}-u_{i+\frac{1}{2}, j}\right)-v_{i-\frac{1}{2}, j+\frac{1}{2}}^{\mathrm{vc}}\left(u_{i-\frac{1}{2}, j+1}-u_{i-\frac{1}{2}, j}\right)\right] \\
& +\eta_{i, j+\frac{1}{2}}^{\mathrm{ns}} v_{i, j+\frac{1}{2}}+\frac{1}{h}\left(p_{i, j+1}-p_{i, j}\right)+\frac{\gamma}{h} \phi_{i, j+\frac{1}{2}}^{\mathrm{ns}}\left(\mu_{i, j+1}-\mu_{i, j}\right) .
\end{aligned}
$$




\section{Now define}

$$
\begin{aligned}
R^{u, \mathrm{e}}:= & -\frac{2}{h^{2}}\left[v_{i+1, j}^{\mathrm{cc}} u_{i+\frac{3}{2}, j}+v_{i, j}^{\mathrm{cc}} u_{i-\frac{1}{2}, j}\right]+\frac{1}{h} p_{i+1, j}+\frac{\gamma}{h} \phi_{i+\frac{1}{2}, j}^{\mathrm{ew}}\left(\mu_{i+1, j}-\mu_{i, j}\right) \\
& -\frac{1}{h^{2}}\left[v_{i+\frac{1}{2}, j+\frac{1}{2}}^{\mathrm{vc}} u_{i+\frac{1}{2}, j+1}+v_{i+\frac{1}{2}, j-\frac{1}{2}}^{\mathrm{vc}} u_{i+\frac{1}{2}, j-1}\right] \\
& -\frac{1}{h^{2}}\left[v_{i+\frac{1}{2}, j+\frac{1}{2}}^{\mathrm{vc}}\left(v_{i+1, j+\frac{1}{2}}-v_{i, j+\frac{1}{2}}\right)-v_{i+\frac{1}{2}, j-\frac{1}{2}}^{\mathrm{vc}}\left(v_{i+1, j-\frac{1}{2}}-v_{i, j-\frac{1}{2}}\right)\right], \\
R^{u, \mathrm{w}}:= & -\frac{2}{h^{2}}\left[v_{i, j}^{\mathrm{cc}} u_{i+\frac{1}{2}, j}+v_{i-1, j}^{\mathrm{cc}} u_{i-\frac{3}{2}, j}\right]-\frac{1}{h} p_{i-1, j}+\frac{\gamma}{h} \phi_{i-\frac{1}{2}, j}^{\mathrm{ew}}\left(\mu_{i, j}-\mu_{i-1, j}\right) \\
& -\frac{1}{h^{2}}\left[v_{i-\frac{1}{2}, j+\frac{1}{2}}^{\mathrm{vc}} u_{i-\frac{1}{2}, j+1}+v_{i-\frac{1}{2}, j-\frac{1}{2}}^{\mathrm{vc}} u_{i-\frac{1}{2}, j-1}\right] \\
& -\frac{1}{h^{2}}\left[v_{i-\frac{1}{2}, j+\frac{1}{2}}^{\mathrm{vc}}\left(v_{i, j+\frac{1}{2}}-v_{i-1, j+\frac{1}{2}}\right)-v_{i-\frac{1}{2}, j-\frac{1}{2}}^{\mathrm{vc}}\left(v_{i, j-\frac{1}{2}}-v_{i-1, j-\frac{1}{2}}\right)\right], \\
R^{v, \mathrm{n}}:= & -\frac{2}{h^{2}}\left[v_{i, j+1}^{\mathrm{cc}} v_{i, j+\frac{3}{2}}+v_{i, j}^{\mathrm{cc}} v_{i, j-\frac{1}{2}}\right]+\frac{1}{h} p_{i, j+1}+\frac{\gamma}{h} \phi_{i, j+\frac{1}{2}}^{\mathrm{ns}}\left(\mu_{i, j+1}-\mu_{i, j}\right) \\
& -\frac{1}{h^{2}}\left[v_{i+\frac{1}{2}, j+\frac{1}{2}}^{\mathrm{vc}} v_{i+1, j+\frac{1}{2}}+v_{i-\frac{1}{2}, j+\frac{1}{2}}^{\mathrm{vc}} v_{i-1, j+\frac{1}{2}}\right] \\
& -\frac{1}{h^{2}}\left[v_{i+\frac{1}{2}, j+\frac{1}{2}}^{\mathrm{vc}}\left(u_{i+\frac{1}{2}, j+1}-u_{i+\frac{1}{2}, j}\right)-v_{i-\frac{1}{2}, j+\frac{1}{2}}^{\mathrm{vc}}\left(u_{i-\frac{1}{2}, j+1}-u_{i-\frac{1}{2}, j}\right)\right], \\
R^{v, \mathrm{~s}}:= & -\frac{2}{h^{2}}\left[v_{i, j}^{\mathrm{cc}} v_{i, j+\frac{1}{2}}+v_{i, j-1}^{\mathrm{cc}} v_{i, j-\frac{3}{2}}\right]-\frac{1}{h} p_{i, j-1}+\frac{\gamma}{h} \phi_{i, j-\frac{1}{2}}^{\mathrm{ns}}\left(\mu_{i, j}-\mu_{i, j-1}\right) \\
& -\frac{1}{h^{2}}\left[v_{i+\frac{1}{2}, j-\frac{1}{2}}^{\mathrm{vc}} v_{i+1, j-\frac{1}{2}}+v_{i-\frac{1}{2}, j-\frac{1}{2}}^{\mathrm{vc}} v_{i-1, j-\frac{1}{2}}\right] \\
& -\frac{1}{h^{2}}\left[v_{i+\frac{1}{2}, j-\frac{1}{2}}^{\mathrm{vc}}\left(u_{i+\frac{1}{2}, j}-u_{i+\frac{1}{2}, j-1}\right)-v_{i-\frac{1}{2}, j-\frac{1}{2}}^{\mathrm{vc}}\left(u_{i-\frac{1}{2}, j}-u_{i-\frac{1}{2}, j-1}\right)\right] .
\end{aligned}
$$

Furthermore, we define

$$
\begin{aligned}
& a^{u, \mathrm{e}}:=2\left(v_{i+1, j}^{\mathrm{cc}}+v_{i, j}^{\mathrm{cc}}\right)+v_{i+\frac{1}{2}, j+\frac{1}{2}}^{\mathrm{vc}}+v_{i+\frac{1}{2}, j-\frac{1}{2}}^{\mathrm{vc}}+\eta_{i+\frac{1}{2}, j}^{\mathrm{ew}} h^{2}, \\
& a^{u, \mathrm{w}}:=2\left(v_{i, j}^{\mathrm{cc}}+v_{i-1, j}^{\mathrm{cc}}\right)+v_{i-\frac{1}{2}, j+\frac{1}{2}}^{\mathrm{vc}}+v_{i-\frac{1}{2}, j-\frac{1}{2}}^{\mathrm{vc}}+\eta_{i-\frac{1}{2}, j}^{\mathrm{ec}} h^{2}, \\
& a^{v, \mathrm{n}}:=2\left(v_{i, j+1}^{\mathrm{cc}}+v_{i, j}^{\mathrm{cc}}\right)+v_{i+\frac{1}{2}, j+\frac{1}{2}}^{\mathrm{vc}}+v_{i-\frac{1}{2}, j+\frac{1}{2}}^{\mathrm{vc}}+\eta_{i, j+\frac{1}{2}}^{\mathrm{ns}} h^{2}, \\
& a^{\mathrm{v}, \mathrm{s}}:=2\left(v_{i, j}^{\mathrm{cc}}+v_{i, j-1}^{\mathrm{cc}}\right)+v_{i+\frac{1}{2}, j-\frac{1}{2}}^{\mathrm{vc}}+v_{i-\frac{1}{2}, j-\frac{1}{2}}^{\mathrm{vc}}+\eta_{i, j-\frac{1}{2}}^{\mathrm{ns}} h^{2} .
\end{aligned}
$$

Putting everything together, the five equations

$$
N_{i \pm \frac{1}{2}, j}^{u}=S_{i \pm \frac{1}{2}, j}^{u} \quad N_{i, j \pm \frac{1}{2}}^{v}=S_{i, j \pm \frac{1}{2}}^{v}, \quad N_{i, j}^{p}=S_{i, j}^{p}
$$


are equivalent to the $5 \times 5$ the matrix equation

$$
\left[\begin{array}{ccccr}
a^{u, \mathrm{e}} h^{-2} & 0 & 0 & 0 & -\frac{1}{h} \\
0 & a^{u, \mathrm{w}} h^{-2} & 0 & 0 & \frac{1}{h} \\
0 & 0 & a^{v, \mathrm{n}} h^{-2} & 0 & -\frac{1}{h} \\
0 & 0 & 0 & a^{v, \mathrm{~s}} h^{-2} & \frac{1}{h} \\
-\frac{1}{h} & \frac{1}{h} & -\frac{1}{h} & \frac{1}{h} & 0
\end{array}\right]\left[\begin{array}{c}
u_{i+\frac{1}{2}, j} \\
u_{i-\frac{1}{2}, j} \\
v_{i, j+\frac{1}{2}} \\
v_{i, j-\frac{1}{2}} \\
p_{i, j}
\end{array}\right]=\left[\begin{array}{c}
S_{i+\frac{1}{2}, j}^{u}-R^{u, \mathrm{e}} \\
S_{i-\frac{1}{2}, j}^{u}-R^{u, \mathrm{w}} \\
S_{i, j+\frac{1}{2}}^{v}-R^{v, \mathrm{n}} \\
S_{i, j-\frac{1}{2}}^{v}-R^{v, \mathrm{~s}} \\
S_{i, j}^{p}
\end{array}\right] .
$$

The Vanka smoothing strategy requires the solution (inversion) of the preceding $5 \times 5$ linear system (B.18), which we represent as $A \mathbf{w}=\mathbf{b}$, for each cell $(i, j), 1 \leq i \leq m$, and $1 \leq j \leq n$. There is a rather simple formula for the solution of system (B.18). See, for example, [31].

\section{References}

[1] A.L. Bertozzi, S. Esedoglu, and A. Gillette. Inpainting of binary images using the CahnHilliard equation. IEEE Trans. Image Proc., 16:285-291, 2007.

[2] H.C. Brinkman. A calculation of the viscous force exerted by a flowing fluid on a dense swarm of particles. Appl. Sci. Res., AI:27-34, 1949.

[3] J.W. Cahn. On spinodal decomposition. Acta Metall., 9:795-801, 1961.

[4] J.W. Cahn, C.M. Elliott, and A. Novick-Cohen. The Cahn-Hilliard equation with a concentration dependent mobility: Motion by minus the Laplacian of the mean curvature. Euro. J. Appl. Math., 7:287-301, 1996.

[5] J.W. Cahn and J.E. Hilliard. Free energy of a nonuniform system. I. Interfacial free energy. J. Chem. Phys., 28:258-267, 1958.

[6] C.M. Elliott and A.M. Stuart. The global dynamics of discrete semilinear parabolic equations. SIAM J. Numer. Anal, 30:1622-1663, 1993.

[7] D. Eyre. Unconditionally gradient stable time marching the Cahn-Hilliard equation. In J. W. Bullard, R. Kalia, M. Stoneham, and L.Q. Chen, editors, Computational and Mathematical Models of Microstructural Evolution, volume 53, pages 1686-1712, Warrendale, PA, USA, 1998. Materials Research Society.

[8] X. Feng. Fully discrete finite element approximations of the Navier-Stokes-Cahn-Hilliard diffuse interface model for two-phase fluid flows. SIAM J. Numer. Anal., 44:1049-1072, 2006.

[9] X. Feng and S.M. Wise. Analysis of a Darcy-Cahn-Hilliard diffuse interface model for the Hele-Shaw flow and its fully discrete finite element approximation. SIAM J. Numer. Anal., 50(3), 1320V1343, 2012.

[10] Z. Hu, S.M. Wise, C. Wang, and J.S. Lowengrub. Stable and efficient finite-difference nonlinear-multigrid schemes for the phase-field crystal equation. J. Comput. Phys., 228:5323-5339, 2009.

[11] D. Kay and R. Welford. A multigrid finite element solver for the Cahn-Hilliard equation. J. Comput. Phys., 212:288-304, 2006.

[12] D. Kay and R. Welford. Efficient numerical solution of Cahn-Hilliard-Navier Stokes fluids in 2D. SIAM J. Sci. Comput., 29:2241-2257, 2007.

[13] J.S. Kim, K. Kang, and J.S. Lowengrub. Conservative multigrid methods for Cahn-Hilliard fluids. J. Comput. Phys., 193:511-543, 2003. 
[14] H.G. Lee, J.S Lowengrub, and J. Goodman. Modeling pinchoff and reconnection in a HeleShaw cell. I. The models and their calibration. Phys. Fluids, 14:492-513, 2002.

[15] H.G. Lee, J.S Lowengrub, and J. Goodman. Modeling pinchoff and reconnection in a HeleShaw cell. II. Analysis and simulation in the nonlinear regime. Phys. Fluids, 14:514-545, 2002.

[16] C. Liu and J. Shen. A phase field model for the mixture of two incompressible fluids and its approximation by a Fourier-spectral method. Physica D, 179:211-228, 2003.

[17] J.S. Lowengrub and L. Truskinovsky. Cahn-Hilliard fluids and topological transitions. Proc. R. Soc. Lond. A, 454:2617-2654, 1998.

[18] W. Ngamsaad, J. Yojina, and W Triampo. Theoretical studies of phase-separation kinetics in a Brinkman porous medium. J. Phys. A: Math. Theor., 43:202001, 2010.

[19] C.W. Oosterlee and F.J. Gaspar. Multigrid relaxation methods for systems of saddle point type. Appl. Numer. Math., 58:1933-1950, 2008.

[20] K. Pham, H.B. Frieboes, V. Cristini, and J. Lowengrub. Predictions of tumour morphological stability and evaluation against experimental observations. J. R. Soc. Interface, 8:16-29, 2011.

[21] J. Shen, C. Wang, X. Wang, and S. Wise. Second-order convex splitting schemes for gradient flows with Ehrlich-Schwoebel-type energy: Application to thin film epitaxy. SIAM J. Numer. Anal., 50:105-125, 2012.

[22] J. Shen and X. Yang. Energy stable schemes for Cahn-Hilliard phase-field model of twophase incompressible flows. Chinese Ann. Math. Series B, 31:743-758, 2010.

[23] J. Shen and X. Yang. Numerical approximations of Allen-Cahn and Cahn-Hilliard equations. Discrete Cont. Dyn. Sys. A, 28:1669-1691, 2010.

[24] J. Shen and X. Yang. A phase-field model and its numerical approximation for two-phase incompressible flows with different densities and viscosities. SIAM J. Sci. Comput., 32:1159$1179,2010$.

[25] U. Trottenberg, C.W. Oosterlee, and A. Schüller. Multigrid. Academic Press, New York, 2005.

[26] S.P. Vanka. Block-implicit multigrid solution of Navier-Stokes equations in primitive variables. J. Comput. Phys., 65:138-158, 1986.

[27] B.P. Vollmayr-Lee and A.D. Rutenberg. Fast and accurate coarsening simulation with an unconditionally stable time step. Phys. Rev. E, 68:066703, 2003.

[28] C. Wang, X. Wang, and S. Wise. Unconditionally stable schemes for equations of thin film epitaxy. Discrete Cont. Dyn. Sys. A, 28:405-423, 2010.

[29] C. Wang and S. Wise. Global smooth solution of modified phase field crystal equation. Methods Appl. Anal., 17:191-212, 2010.

[30] C. Wang and S. Wise. An energy stable and convergent finite-difference scheme for the modified phase field crystal equation. SIAM J. Numer. Anal., 49:945-969, 2011.

[31] P. Wesseling. An Introduction to Multigrid Methods. R.T. Edwards, Philadelphia, 2004.

[32] S.M. Wise. Unconditionally stable finite difference, nonlinear multigrid simulation of the Cahn-Hilliard-Hele-Shaw system of equations. J. Sci. Comput., 44:38-68, 2010.

[33] S.M. Wise, J.S. Lowengrub, and V. Cristini. An adaptive algorithm for simulating solid tumor growth using mixture models. Math. Comput. Model., 53:1-20, 2011.

[34] S.M. Wise, J.S. Lowengrub, H.B. Frieboes, and V. Cristini. Three-dimensional multispecies nonlinear tumor growth - I model and numerical method. J. Theor. Biol., 253:524-543, 2008.

[35] S.M. Wise, C. Wang, and J.S. Lowengrub. An energy stable and convergent finite-difference scheme for the phase field crystal equation. SIAM J. Numer. Anal., 47:2269-2288, 2009. 